\title{
XXXII. Radiation and electromagnetic theory
}

\section{R. Hargreaves}

To cite this article: R. Hargreaves (1905) XXXII. Radiation and electromagnetic theory , Philosophical Magazine Series 6, 9:51, 313-350, DOI: 10.1080/14786440509463285

To link to this article: http://dx.doi.org/10.1080/14786440509463285

$$
\text { 册 Published online: } 15 \text { Apr } 2009 .
$$

Submit your article to this journal

Џ Article views: 5

Q View related articles $\sqsubset$ 
The first is effected by putting $l^{2} n^{2}=2 \mathrm{R} r+r^{2}$, and the second by making the selte-induction a maximum. We shall now see how the third can be effected. The difference in phase when $\frac{\mathrm{P}_{1}-\mathrm{P}_{2}}{\mathrm{P}_{1}+\mathrm{P}_{2}}$ is a maximum is
given by equation (5).

Replacing $\sin 2 \alpha$ and $\cos 2 \alpha$ by their corresponding values, we get

$$
\begin{aligned}
\frac{\mathrm{P}_{1}-\mathrm{P}_{2}}{\mathrm{P}_{1}+\mathrm{P}_{2}} & =\frac{2 \mathrm{R} l n(\mathrm{R}+r)}{\sqrt{\left(2 \mathrm{R} r+r^{2}\right)\left\{4 \mathrm{R}^{2} l^{2} n^{2}+\left(2 \mathrm{R} r+r^{2}+l^{2} n^{2}\right)^{2}\right\}}} \\
& =\frac{2 \mathrm{R}(\mathrm{R}+r)}{\sqrt{2 \mathrm{R} r+r^{2}} \sqrt{\left(2 \mathrm{R}+r^{2}+r^{2}+\left(\frac{2 \mathrm{R} r+r^{2}}{l n}-l n\right)^{2}\right.}}
\end{aligned}
$$

This is a maximum when $l^{2} n^{2}=2 \mathrm{R} r+r^{2}$, that is when the difference in phase is $90^{\circ}$; and it will be found that the selfinduction can be altered to a considerable extent above or below this value, without affecting the control materially.

Taking into consideration, then, the fact that the selfinduction is not constant, but varies to a certain extent with the current, and that in any case it is only possible to measure it approximately, it seems advisable to adjust it in such a manner as to make $l^{2} n^{2}$ as nearly as possible equal to $2 \mathrm{R} r+r^{2}$.

This allows a large margin either way without any appreciable loss of control, damps down the surging currents, and finally avoids all fluctuation in the external circuit by keeping constant the total power given out by the two alternators.

I have to thank Professor Greenhill for suggestions and for verification of the formulas involved.

XXXII. Radiation and Electromagnetic Theory. By R. Hargreaves*.

\$1-3. Radiation formulæ connected with change of period in reflexion at a moving surface. Connexion with laws of Stefan and Wien.

\$4-8. Refraction. Formula for pressure.

\$ 9-12. Electromagnetic waves viewed from a moving standpoint. Fresnel's formula. A berration.

13-16. Reflexion and refraction. Application to formulæ in first section. Calculation of pressure, \&c

$\$ 17-19$. Introduction of charge and current into the equations. Electromagnetic momentum or momentum of radiation.

REFLEXION at a moving surface has certain distinct 1 features: the angles of incidence and reflexion are unequal, the periods of the incident and reflected waves are different. At the same time energy is converted to mechanical work by the action of pressure on a moving surface.

* Communicated by the Author. 
The special feature of the transformation is the relation of the formula for Doppler's effect to the mode in which allowance is made for this conversion of energy.

The translation in the first section is not general, but it will] appear in $\$ 7$ that when reflexion only is in question it is not necessary to consider other components.

$\$ 1$. First take the case of reflexion at a perfectly reflecting plane surface, which is retreating directly from the free æther space with uniform velocity $w$. If $p\{t-(l x+m y+n z) / V\}$ is the argument of an original, and $p^{\prime}\left\{t-\left(l^{\prime} x+m^{\prime} y-n^{\prime} z\right) / \mathrm{V}\right\}$ that of the reflected wave, the kinematical part of the law of reflexion consists in the identification of arguments at the moving surface, $i$. e., for a value $z=z_{0}+w t$ : the identification to hold for all values of $t, x$, and $y$. The conditions are

$$
p l=p^{\prime} l, \quad p m=p^{\prime} m^{\prime}, \quad p(1-w n / V)=p^{\prime}\left(1+w n^{\prime} / V\right) .
$$

The former pair may be replaced by $p^{2}\left(1-n^{2}\right)=p^{\prime 2}\left(1-n^{\prime 2}\right)$, and the law of reflexion is

$$
\left(1-n^{2}\right) /(1-w n / \mathrm{V})^{2}=\left(1-n^{2}\right) /\left(1+w n^{\prime} / \mathrm{V}\right)^{2}, \quad .
$$

with a common value $\phi$ for an azimuthal angle involved in

$$
\begin{array}{ll}
l=\sqrt{1-n^{2}} \cos \phi, m=\sqrt{1-n^{2}} \sin \phi, l & =\sqrt{1-n^{\prime 2}} \cos \phi, \\
& m^{\prime}=\sqrt{1-n^{\prime 2}} \sin \phi .
\end{array}
$$

$$
\text { or } \begin{aligned}
(\mathrm{V} n-w) d n /(1-w n / \mathrm{V})^{3}=\left(\mathrm{V} n^{\prime}+w\right) d n^{\prime} /\left(1+w n^{\prime} / \mathrm{V}\right)^{3}, \\
p^{3}(\mathrm{~V} n-w) d n=p^{\prime 3}\left(\mathrm{~V} n^{\prime}+w\right) d n^{\prime},
\end{aligned}
$$

and multiplication by the last of (1) gives

$$
p^{4}(\mathrm{~V} n-w)(1-w n / \mathrm{V}) d n=p^{\prime 4}\left(\mathrm{~V} n^{\prime}+w\right)\left(1+w n^{\prime} / \mathrm{V}\right) d n^{\prime},
$$

a formula of fundamental importance. The law of reflexion may be put in linear form, viz. with $r=w / \mathrm{V}$,

$$
\frac{\mathrm{V} n-w}{\mathrm{~V}-w n}=\frac{\mathrm{V} n^{\prime}+w}{\mathrm{~V}+w n^{\prime}}, \text { or } \frac{n-r}{1-r n}=\frac{n^{\prime}+r}{1+r n^{\prime}}, .
$$

which follows from (2) by using

$$
1-\left(\frac{n-r}{1-r n}\right)^{2}=\frac{\left(1-r^{2}\right)\left(1-n^{2}\right)}{(1-r n)^{2}} .
$$

We may note also the relations

$$
p(\mathrm{~V} n-w)=p^{\prime}\left(\mathrm{V} n^{\prime}+w\right), \text { and } p^{2} d n=p^{\prime 2} d n^{\prime} . .
$$


It is proposed to obtain a relation between mean values of $p^{4}$ and $p^{\prime 4}$ independent of the angle of incidence by integrating the two sides of (3) with regard to $n$ and $n^{\prime}$ respectively, while leaving the mean values $p^{4}$ and $p^{\prime 4}$ outside the sign of integration. We may dispense with a separate notation, and describe the resulting relation between $p$ and $p^{\prime}$ as a collective relation: this is regarded as appropriate to two streams of radiation incident and reflected, the former evenly distributed as regards direction. The proper limits for $n$ are $r$ and 1 , for $n^{\prime}$ they are $-r$ and 1 ; it is evident that a wave for which $n<r$ fails to reach the surface, and $n=r$ corresponds to $n^{\prime}=-r$ by (4). The result of the integration is

$$
p^{4}(1-r)^{3}\left(1+\frac{r}{3}\right)=p^{\prime 4}(1+r)^{3}\left(1-\frac{r}{3}\right) . . .
$$

Both in (3) and in (6) $p^{4}$ and $p^{4}$ may be replaced by $p^{3} d p$ and $p^{\prime 3} d p^{\prime}$, and for (5) may be written $p d p d n=p^{\prime} d p^{\prime} d n^{\prime}$. Since the wave-length $\lambda=2 \pi V / p, \lambda^{-1}$ and $\lambda^{\prime-1}$ may take the places of $p$ and $p^{\prime}$, or $d \lambda / \lambda^{5}$ and $d \lambda^{\prime} / \lambda^{15}$ those of $p^{3} d p$ and $p^{\prime 3} d p^{\prime}$.

\$2. Analytically the process is that of transformation of a double integral with subsequent integration with regard to one variable on each side. A continuous range of values for $p$ and $p^{\prime}$ is assumed, and if the range is limited the relation is not applicable within a certain margin near the limit.

The form of (3) points to its interpretation as an equation for the transfer of energy by reflexion, with an allowance for the work done by pressure and for the filling of the new space opened out by the motion, with energy-content. In Wien's theory energy-content is proportional to $d \lambda \lambda^{5} \mathrm{mul}$ tiplied by a function of $\lambda \theta$, or to $p^{3} d p$ multiplied by a function of $p / \theta$, when $p$ is variable. If in the two streams $p / \theta=p^{\prime} / \theta^{\prime}$ the energies are as $p^{3} d p: p^{\prime 3} d p^{\prime}$ or as $p^{4}: p^{4}$; and the above equation (6) connects the energy-content in a stream of incident radiation with that in the reflected stream. The possibility of writing $p / \theta=p^{\prime} / \theta^{\prime}$ depends on the invariable collective relation between $p$ and $p^{\prime}$. On the other hand, the notion of temperature as a general energy parameter which is implied in Wien's function, is not admissible for the individual wave, and its application seems to demand a preliminary treatment of a collective character.

If $\chi$ is used for the energy-content belonging to radiation proceeding in one sense (directions covering a hemisphere), and is evenly distributed, the section between the cones $n$ and $n+d n$ is $\chi^{d n}$. We take it that $\chi$ and $\chi^{\prime}$ may replace 
$p^{4}$ and $p^{4}$ in the integrated form (6), and consider the interpretation. With odd and even powers of $r$ separated, the exact formula is

and if $r$ is small,

$$
\left(\chi-\chi^{\prime}\right)\left(1+2 r^{2}-\frac{r^{4}}{3}\right)=\frac{8 r}{3}\left(\chi+\chi^{\prime}\right)
$$

$$
\frac{\mathrm{V}}{2}\left(\chi-\chi^{\prime}\right)=w\left(\chi+\chi^{\prime}\right)+\frac{w}{3}\left(\chi+\chi^{\prime}\right), \ldots .
$$

which gives a rough idea of the meaning. At a fixed plane parallel to the moving reflector, the left-hand member gives the excess of the rate of passage of the outgoing radiation above the returning radiation (radiation formula $\chi \int_{0}^{1} V n d n$ ) ; the first term on the right is the rate at which energy-content is supplied to the new space, and the second term is the rate of working of pressure (formula $\int_{0}^{1} n$. $v 0 n \cdot \chi^{d n}$ ). When $r$ is small, (7) gives the ratio $\chi: \chi^{\prime}$ correctly to the second order; but when $r$ approaches 1 (or $w$ approaches $V$ ) the difference is important, and the exact formula makes $\chi^{\prime}$ vanish with $1-r$.

Electromagnetic theory gives the more detailed interpretation through the form

$\chi \int \nabla n d n=\chi \int(\mathrm{V} n-w)(1-r n) d n+w \chi \int(1-r n) d n+w \chi \int n^{2} d n$.

The radiation across a fixed plane provides (1) the exchange value, or flux at a moving surface, with (2) the energy-content proper to a moving standpoint for the space opened out, and (3) the work done by pressure.

The formula for pressure in reflexion is

$$
\chi \int_{r}^{1} n^{2} d n+\chi^{\prime} \int_{-r}^{1} n^{\prime 2} d n^{\prime} \quad \text { or } \quad \frac{\chi}{3}\left(1-r^{3}\right)+\frac{\chi^{\prime}}{3}\left(1+r^{3}\right),
$$

or

$$
\frac{1}{3}\left(\chi+\chi^{\prime}\right)-\frac{r^{3}}{3}\left(\chi-\chi^{\prime}\right)
$$

and the exact division into parts with the meaning assigned in (7) is

$$
\begin{array}{r}
\frac{\mathrm{V}}{2}\left(\chi-\chi^{\prime}\right)=w\{ \\
\left.x+\chi^{\prime}-r\left(1-\frac{r^{2}}{2}\right)\left(\chi-\chi^{\prime}\right)\right\} \\
+\frac{w}{3}\left\{\chi+\chi^{\prime}-r^{3}\left(\chi-\chi^{\prime}\right)\right\}
\end{array}
$$

$\S 3$. The collective formula presupposes a continuous range 
of values of $p$; if the range is limited, then a margin of values of $p^{\prime}$ will be imperfectly represented, or the relation between $p$ and $p^{\prime}$ ceases to have the uniform character given above within a margin at either extreme. The extent of such margin depends on $r$ or $w / \mathrm{V}$, and when $r$ is small is inappreciable; but is not so if $r$ is finite. The conclusions drawn as to the fourth power of temperature require the range of $p$ to be made sufficiently wide to ensure this penumbral effect being of no importance, but need not be infinite unless $w=\mathrm{V}$.

In connexion with this question of the fourth power, if we look back to the construction of (3) the use of

or $\quad p^{2}(\mathrm{~V} n-w) d p d n=p^{\prime 2}\left(\mathrm{~V} n^{\prime}+w\right) d p^{\prime} d n^{\prime}$

seems to be essential, the further subdivision into

$$
p d p d n=p^{\prime} d p^{\prime} d n^{\prime} \text { and } p(\mathrm{~V} n-w)=p^{\prime}(\mathrm{V} n+w)
$$

not being possible in refraction. As a matter of analysis, a more general collective relation, involving transit with some loss or modification, would be got by multiplying the above by an arbitrary function of

$$
p(1-q n) \quad \text { or } \quad p^{\prime}\left(1+q n^{\prime}\right) .
$$

When the object is to allow for work done by pressure it is prima facie reasonable to make the work depend directly on won the component of translation along the wave-normal, and use the linear factor. In this way the theory is brought into relation with the laws of Stefan and Wien; but the point will be mentioned later.

In reflexion two of the factors $p$ in $p^{4}$ are connected with the angular correspondence in the original and reflected streams, the other two are connected with the relative velocities $V n-w$ and $V-w n$, the former a velocity of transit, the latter connected with change of period. When we pass to the collective relation this is true as to the source of the power in $\theta^{4}$. In effect radiation being a question of exchange, its law depends on the degree of freedom for exchange: for the analogues in two dimensions and in one dimension the appropriate powers are the cube and the square.

For two dimensions (2) is correct, but (3) suggests a wrong angular element: the element is $d \theta$ (not $\sin \theta d \theta, i$. e. $\sin \theta d \theta d \phi$ with $d \phi$ discarded), and

$$
d n: d n^{\prime}=d \theta \sqrt{1-n^{2}}: d \theta^{\prime} \sqrt{1-n^{\prime 2}}=p d \theta: p^{\prime} d 6^{\prime} ;
$$

so that for (3) should be written

$p^{3} \int(\mathrm{V} \cos \theta-w)(1-r \cos \theta) d \theta=p^{\prime 3} \int\left(\mathrm{V} \cos \theta^{\prime}+w\right)\left(1+r \cos \theta^{\prime}\right) d \theta^{\prime}$. 
The integrated relation is

$$
\begin{aligned}
p^{3}\left[r^{\prime}\right. & \left.\left(1+r^{2}\right)-\frac{r}{2}\left(r r^{\prime}+3 \arcsin r^{\prime}\right)\right] \\
\quad=p^{\prime 3} & {\left[r^{\prime}\left(1+r^{2}\right)+\frac{r}{2}\left(3 \pi-\arcsin r^{\prime}-r r^{\prime}\right)\right], }
\end{aligned}
$$

where $r^{\prime}=\sqrt{1-r^{2}}$. In the limit when $r^{\prime}$ is small or $w$ nearly $=V, p^{\prime 3} / p^{3}$ varies as $r^{\prime 5}$ or $\left(1-r^{2}\right)^{5 / 2}$. The approximate form analogous to (7) is

$$
\frac{2 V}{\pi}\left(\chi-\chi^{\prime}\right)=w\left(\chi+\chi^{\prime}\right)+\frac{w}{2}\left(\chi+\chi^{\prime}\right) ;
$$

and the exact estimate of pressure is

$$
\frac{\chi}{\pi}\left(\arcsin r^{\prime}+r r^{\prime}\right)+\frac{\chi^{\prime}}{\pi}\left(\pi-\arcsin r^{\prime}+r r^{\prime}\right) .
$$

In one dimension we have the single relation

$$
p(1-w / V)=p^{\prime}(1+w / V),
$$

which must be duplicated in the form

$$
p^{2}(\mathrm{~V}-w)(1-w / \mathrm{V})=p^{\prime 2}(\mathrm{~V}+w)(1+w / \mathrm{V}),
$$

to show the change of period and velocity of transit. No integration occurs, $\chi$ and $\chi^{\prime}$ are written for $p^{2}$ and $p^{\prime 2}$, and the pressure is $\chi+\chi^{\prime}$.

The special features in the comparison of the cases for different dimensions are :- for radiation formulæ

$$
\frac{\mathrm{V} \chi}{2}, \frac{2 \mathrm{~V} \chi}{\pi}, \text { and } \mathrm{V} \chi
$$

for the limits when $w=\mathrm{V}$ or $r=1, p^{\prime}$ varies as $(1-r)^{3 / 4}$, $(1-r)^{5 / 6}, 1-r$; and $\chi^{\prime}$ as $(1-r)^{3},(1-r)^{5 / 2},(1-r)^{2}$.

$\S 4$. When refraction accompanies reflexion there are two channels into which energy is directed, and the two transformations concerned are only part of the problem : the partition of energy requires distinctive optical theory. The special features affecting the transformation for refraction are (1) a modified velocity of propagation in the dielectric, and (2) the necessity for interpretation with reference to the moving standpoint of the dielectric.

At first we use an argument referred to the same coordinates as the original wave, take $p^{\prime \prime}$ for period number, and suppose $\Omega$ the velocity of propagation to depend on 
$l^{\prime \prime} u+m^{\prime \prime} v+n^{\prime \prime} w$ or $\mathrm{U}^{\prime \prime}$. The comparison of arguments gives

$$
\left.\begin{array}{c}
\frac{p l}{\mathrm{~V}}=\frac{p^{\prime \prime} l^{\prime \prime}}{\Omega}, \frac{p m}{\mathrm{~V}}=\frac{p^{\prime \prime} m^{\prime \prime}}{\Omega}, p\left(1-\frac{w n}{\mathrm{~V}}\right)=p^{\prime \prime}\left(1-\frac{w n^{\prime \prime}}{\Omega}\right) ; \\
p\left(1-\frac{\mathrm{U}}{\mathrm{V}}\right)=p^{\prime \prime}\left(1-\frac{\mathrm{U}^{\prime \prime}}{\Omega}\right),
\end{array}\right\}
$$

follows by combining the other ratios. The correspondence of $p$ and $p^{\prime \prime}$ is the same whether deduced from agreement at a moving point or surface. If the ratio $p: p^{\prime \prime}$ is eliminated,

or

$$
\left.\begin{array}{c}
\left(\mathrm{L}-n^{2}\right) /(\mathrm{V}-w n)^{2}=\left(1-n^{\prime 2}\right) /\left(\Omega-w n^{\prime \prime}\right)^{2} \\
\left(1-n^{2}\right) /(\mathrm{V}-\mathrm{U})^{2}=\left(1-n^{\prime \prime}\right) /\left(\Omega-\mathrm{U}^{\prime \prime}\right)^{2}
\end{array}\right\}
$$

An azimuthal angle $\phi\left(l=\sqrt{1-n^{2}} \cos \phi\right)$ is the same for both waves, this fact and (10) together constituting the law of refraction. Since $\phi$ is the same for both waves it may be ignored in forming the differentials of $(10)$, and we take account of the dependence of $l^{\prime \prime}, m^{\prime \prime}$ on $n^{\prime \prime}$ by writing

Hence

$$
\frac{d l^{\prime \prime}}{l^{\prime \prime}}=\frac{-n^{\prime \prime} d n^{\prime \prime}}{1-n^{\prime 2}}=\frac{d m^{\prime \prime}}{m^{\prime \prime}} \text {. }
$$

or

$$
d \Omega=\Sigma \frac{d \Omega}{d l^{\prime}} d l^{\prime \prime}=\frac{d \Omega}{d \mathrm{U}^{\prime \prime}} \Sigma u d l^{\prime \prime},
$$

$\left(1-n^{\prime 2}\right) d \Omega=\frac{d \Omega}{d \mathrm{U}^{\prime \prime}}\left(w-n^{\prime \prime} \mathrm{U}^{\prime \prime}\right) d n^{\prime \prime}$,

and the differential of $(10)$ yields

$$
\frac{(\mathrm{V} n-w) d n}{(\mathrm{~V}-\mathrm{U})^{3}}=\frac{\left\{n^{\prime \prime}\left(\Omega-\mathrm{U}^{\prime \prime} \frac{d \Omega}{d \mathrm{U}^{\prime \prime}}\right)-w\left(1-\frac{d \Omega}{d \mathrm{U}^{\prime \prime}}\right)\right\} d n^{\prime \prime}}{\left(\Omega-\mathrm{U}^{\prime \prime}\right)^{3}} .
$$

Either form of (10) may be used, and the denominators here may be replaced by $(\mathrm{V}-w n)^{3}$ and $\left(\Omega-w n^{\prime \prime}\right)^{3}$. The numerators contain a differential and a velocity of transit, and $d \phi$ may be appended to each side. [If $\Omega$ is defined as a function of $l^{\prime \prime} m^{\prime \prime} n^{\prime \prime}$ without reference to $U^{\prime \prime}$, the transitform is

$$
\left.n^{\prime \prime}\left(\Omega-\Sigma l^{\prime \prime} \frac{d \Omega}{d l^{\prime \prime}}\right)+\frac{d \Omega}{d n^{\prime \prime}}-w .\right]
$$

With Fresnel's modification, which is used throughout,

$$
\Omega=\frac{V}{\mu}+U^{\prime \prime}\left(1-\frac{1}{\mu^{2}}\right)
$$


and the above takes the simpler form

$$
\frac{(\mathrm{V} n-w) d n}{(\mathrm{~V}-\mathrm{U})^{3}}=\frac{\left(\frac{\mathrm{V} n^{\prime \prime}}{\mu}-\frac{w}{\mu^{2}}\right) d n^{\prime \prime}}{\left(\frac{\mathrm{V}}{\mu}-\frac{\mathrm{U}^{\prime \prime}}{\mu^{2}}\right)^{3}} ; .
$$

the transit-velocity, or component of ray-velocity, depending only on $n^{\prime \prime}$. Introducing the fourth powers of $p$ and $p^{\prime \prime}$,

$$
p^{4} \mathrm{~V}^{-4}(\mathrm{~V} n-w)(\mathrm{V}-\mathrm{U}) d n=p^{\prime \prime 4} \Omega^{-4}\left(\frac{\mathrm{V} n^{\prime \prime}}{\mu}-\frac{w}{\mu^{2}}\right)\left(\frac{\mathrm{V}}{\mu}-\frac{\mathrm{U}^{\prime \prime}}{\mu^{2}}\right) d n^{\prime \prime} \text {. }
$$

With the wave-length as variable, since $p^{\prime \prime} / \Omega=2 \pi \lambda^{\prime \prime}$,

and

$$
\begin{aligned}
\lambda: \lambda^{\prime \prime} & =l: l^{\prime \prime}=m: m^{\prime \prime}=\mathrm{V}-w n: \Omega-w n^{\prime \prime} \\
& =\mathrm{V}-\mathrm{U}: \Omega-\mathrm{U}^{\prime \prime}=\mathrm{V}-\mathrm{U}: \frac{\mathrm{V}}{\mu}-\frac{\mathrm{U}^{\prime \prime}}{\mu^{2}},
\end{aligned}
$$

$\lambda^{-4}(\mathrm{~V} n-w)(\mathrm{V}-\mathrm{U}) d n=\lambda^{\prime \prime-4}\left(\frac{\mathrm{V} n^{\prime \prime}}{\mu}-\frac{w}{\mu^{2}}\right)\left(\frac{\mathrm{V}}{\mu}-\frac{\mathrm{U}^{\prime \prime}}{\mu^{2}}\right) d n^{\prime \prime}$.

We now give a transformation of a different kind, in which the ray-cosines define direction, and a period-number is used which has reference to a moving standpoint. For free æther this is $\sigma=p(1-\mathrm{U} / \mathrm{V})$, and for the dielectric $\sigma^{\prime \prime}=p^{\prime \prime}\left(1-\mathrm{U}^{\prime \prime} / \Omega\right)$, and by $(9) \varpi=\sigma^{\prime \prime}$. With (11) the transfer from $p$ to $\varpi$ or $\varpi^{\prime \prime}$ is given by

$$
\begin{gathered}
p^{4}(\mathrm{~V} n-w)(1-\mathrm{U} / \mathrm{V}) d n / \mathrm{V}^{3}=\sigma^{4}(\mathrm{~V} n-w) d n /(\mathrm{V}-\mathrm{U})^{3} \\
=\sigma^{\prime \prime}\left(\frac{\mathrm{V} n^{\prime \prime}}{\mu}-\frac{w}{\mu^{2}}\right) d n^{\prime \prime} /\left(\frac{\mathrm{V}}{\mu}-\frac{\mathrm{U}^{\prime \prime}}{\mu^{2}}\right)^{3} .
\end{gathered}
$$

Ray-cosines $\left(l_{r} m_{r} n_{r}\right)$ are connected with the wave-normal cosines by the scheme

$\left.\begin{array}{c}\mathrm{V}_{r} l_{r}=\mathrm{V} l-u, \quad \mathrm{~V}_{r} m_{r}=\mathrm{V}_{m}-v, \quad \mathrm{~V}_{r} n_{r}=\mathrm{V} n-w, \\ \mathrm{~V}_{r}{ }^{2}=\mathrm{V}^{2}-2 \mathrm{VU}+\Sigma u^{2}, \quad \mathrm{~V}_{r}{ }^{2}+2 \mathrm{~V}_{r} \mathrm{U}_{r}=\mathrm{V}^{2}-\Sigma u^{2}, \\ \mathrm{~V}_{r} \Sigma_{r} l_{r} l=\mathrm{V}-\mathrm{U}, \mathrm{V} \Sigma l_{r} l=\mathrm{V}_{r}+\mathrm{U}_{r}=\sqrt{\mathrm{V}^{2}-\Sigma u^{2}+\mathrm{U}_{r}^{2}}\end{array}\right\}$,

$\mathrm{V}$, being a ray-velocity and $\mathrm{U}_{\boldsymbol{r}}$ standing for $\Sigma l_{\boldsymbol{r}} u$. In a dielectric $\frac{V}{\mu}, \frac{u}{\mu^{2}}, \ldots$ take the places of $\mathrm{V}, u, \ldots$ The radical is the wave-velocity in the direction of the ray. Comparing $\sqrt{\sqrt{V^{2}}-\Sigma u^{2}+U_{r}^{2}}-U_{r}$ and $\sqrt{\mathrm{V}^{2}-\Sigma u^{2}+U_{r}^{2}}+U_{r}$, which are the numerical values of $\nabla_{r}$, with $\mathrm{V}-\mathrm{U}$ and $\mathrm{V}+\mathrm{U}$ belonging to translation with or against the wave, the radical 
plays a part corresponding to that of $V$. For the transformation use the variables $\left(n_{r} \phi_{r}\right),(n \phi)$, where $\tan \phi_{r}=m_{r} / l_{r}$, $\tan \phi=m / l$; the Jacobian after some reduction gives

Hence

$$
\mathrm{V}_{r}^{3}\left(\frac{d n_{r}}{d n} \frac{d \phi_{r}}{d \phi}-\frac{d n_{r}}{d \phi} \frac{d \phi_{r}}{d n}\right)=\nabla^{2}(\mathrm{~V}-\mathrm{U})
$$

$$
\begin{array}{r}
\varpi^{4}(\mathrm{~V} n-w) d n d \phi /(\mathrm{V}-\mathrm{U})^{3}=\varpi^{4} V_{r} n_{r} d n d \phi /(\mathrm{V}-\mathrm{U})^{3} \\
=\mathrm{V}^{2} \varpi^{4} n_{r} d n_{r} d \phi_{r} /\left\{\nabla^{2}-\Sigma u^{2}+\mathrm{U}_{r}^{2}\right\}^{2},
\end{array}
$$

since by (16) $\quad V(\nabla-U)=V_{r} \sqrt{V^{2}-\Sigma u^{2}+\mathrm{U}_{r}{ }^{2}}$.

Apply to the third member of (15) with $d \phi$ appended a like transformation, and we get

$$
\begin{gathered}
p^{4}(n-w / \mathrm{V})(1-\mathrm{U} / \mathrm{V}) d n d \phi=\varpi^{4} n_{r} d n_{r} d \phi_{r} /\left\{1-\Sigma u^{2} / V^{2}+\mathrm{U}_{r}^{2} / \nabla^{2}\right\}^{2} \\
=\mu^{2} \varpi^{\prime \prime} n_{r}{ }^{\prime \prime} d n_{r}{ }^{\prime \prime} d \phi_{r}{ }^{\prime \prime} /\left\{1-\Sigma^{2} / \mu^{2} V^{2}+U_{r}^{\prime \prime 2} / \mu^{2} V^{2}\right\}^{2} .
\end{gathered}
$$

$\$ 5$. Consider first the statical case, in which $\approx$ coalesces with $p$. It is then indifferent whether $p^{\prime \prime}$ or $\lambda^{\prime \prime}$ is used as variable, and the form of equations (12) and (14) suggests for the ratio of energy-contents in the two media

$$
\chi: \chi^{\prime \prime}=\nabla / \lambda^{4}: \nabla^{\prime \prime} / \lambda^{\prime \prime 4}=p^{4} / \nabla^{8}: p^{\prime \prime 4} / \nabla^{\prime 3}, .
$$

with $V^{\prime \prime}$ for $V / \mu$ : since $p=p^{\prime \prime}$ or $\mu \lambda^{\prime \prime}=\lambda, \chi^{\prime \prime}=\mu^{3} \chi$. We may then write $p / \theta=p^{\prime \prime} / \theta^{\prime \prime}$ or its equivalent $\lambda \theta=\mu \lambda^{\prime \prime} \theta^{\prime \prime}$. This is consistent with the use of Wien's formula in the shape

$$
\nabla^{\prime \prime-3} p^{L / 3} d p^{\prime \prime} f\left(p^{\prime \prime} / \theta^{\prime \prime}\right) \text { or } \nabla^{\prime \prime} \lambda^{\prime \prime-5} d \lambda^{\prime \prime} f\left(V^{\prime \prime} / \lambda^{\prime \prime} \theta^{\prime \prime}\right) \text {, }
$$

for the result of integration through an infinite range of $p^{\prime \prime}$ or $\lambda^{\prime \prime}$ is proportional to $\theta^{\prime \prime 4} / \nabla^{\prime \prime 3}$, and the condition of statical exchange $V n\left(\theta^{4} / V^{3}\right) d n=\nabla^{\prime \prime} n^{\prime \prime}\left(\theta^{\prime \prime 4} / V^{1 / 3}\right) d n^{\prime \prime}$ makes $\theta=\theta^{\prime \prime}$ follow from $n d n / V^{2}=n^{\prime \prime} d n^{\prime \prime} / V^{\prime \prime 2}$.

When there is motion the use of $\left(p, p^{\prime}\right)$ or $\left(\lambda, \lambda^{\prime}\right)$ in reffexion is indifferent. But there is this real difference between the original and reflected waves on the one hand, and the refracted wave on the other, that for the former the moving standpoint represents a momentary phase, while for the latter it is permanent, and moreover is that with reference to which we naturally seek to interpret matters. Now an argument $\Sigma l x^{\prime}-\mathrm{V} t$ becomes $\Sigma l x-(\mathrm{V}-\mathrm{U}) t$, when by the use of $x^{\prime}=x+u t, \ldots$ a change is made to a moving standpoint; the change alters the period not the wave-length, and this is equally true if the variable velocity $\Omega$ is used instead of $\nabla$.

Phil. Mag. S. 6. Vol. 9. No. 51. March $1905 . \quad$ Y 
Thus if the formula in $\lambda^{\prime \prime}$ is used it is clear that

$$
\left(\frac{\mathrm{V}}{\mu}-\frac{\mathrm{U}^{\prime \prime}}{\mu^{\psi}}\right) \lambda^{\prime \prime-4} d n^{\prime \prime} \text { not } \frac{\mathrm{V}}{\mu} \lambda^{\prime \prime-4} d n^{\prime \prime}
$$

must be proportional to energy-content, otherwise there would be nothing to mark the moving standpoint. This agrees with a formula used above (8) for the energycontent in space opened out by the motion. The formula uses wave variables for direction.

If energy-content were taken proportional to $\frac{\mathrm{V}}{\mu} \lambda^{\prime \prime-4} d n^{\prime \prime}$, then the term in $U^{\prime \prime}$ would necessarily be interpreted as on the other side of the equation, and would imply a backpressure on the rther caused by the refracted ray (v. infra).

The formula in $\approx$ has no term which could be interpreted in this manner, and a special feature is that $(u v w)$ only occur in second order terms. 'The use of $\approx$ and ray-cosines both imply a new standpoint; the fact that $w$ and $\sigma^{\prime \prime}$ are equal makes the change of standpoint rather than that of medium the important fact in (17).

$\S 6$. The collective relations when reflexion and refraction are combined must take account of the proportion in which the radiation is divided into two streams, a proportion varying with the angle of incidence.and with the plane of polarization. For the present we denote by $\mathrm{A}^{2}$ and $\mathrm{B}^{2}$ the fractions (sum unity) of the original energy which undergo the respective transformations; the value of $\mathrm{A}^{2}$ is given by electromagnetic theory, and will be found to depend on $(u v w)$ as well as on the angular variables. It is proposed to weight the equations of transformation with $\mathrm{A}^{2}$ for the reflected and $\mathrm{B}^{2}$ for the refracted section; and then to integrate them in a manner which will clear the results of all reference to particular waves. The relations involve $n$ and $\phi$ (through $\mathrm{U}$ ), and $\mathrm{A}^{2}$ also involves an angle defining the plane of polarization. But if we suppose $\mathrm{A}^{z}$ to stand for the mean with regard to this last angle, the plane of polarization taking all possible positions for each wave-argument, then only $n$ and $\phi$ need appear, i.e.

$$
\begin{array}{r}
\begin{aligned}
p^{4} \iint \mathrm{A}^{2}(\mathrm{~V} n-w) & (\mathrm{I}-\mathrm{U} / \mathrm{V}) d n d \phi \\
& =p^{\prime 4} \iint \mathrm{A}^{2}\left(\mathrm{~V} n^{\prime}+w\right)\left(1-\mathrm{U}^{\prime} / \mathrm{V}\right) d n^{\prime} d \phi
\end{aligned} \\
\begin{aligned}
p^{4} \iint \mathrm{B}^{2}(\mathrm{~V} n-w)(1-\mathrm{U} / \mathrm{V}) d n d \phi \\
=
\end{aligned} \\
\text { and the sum } \iint \mathrm{B}^{2} \mathrm{~V} n_{r} d n_{r} d \phi_{r} /\left(1-\Sigma u^{2} / \mathrm{V}^{2}+\mathrm{U}_{r}^{2} / \mathrm{V}^{2}\right)^{2} \\
\begin{array}{r}
p^{4} \iint(\mathrm{V} n-w)(1-\mathrm{U} / \mathrm{V}) d n d \phi \\
=
\end{array}
\end{array}
$$


is the equation for flux of energy. [The formula for or $\sigma^{\prime \prime}$ may be used, $v$. infra.j The terms representing a rate of mechanical working are

$$
p^{4} \iint n \mathrm{U} d n d \phi-p^{\prime 4} \iint \mathrm{A}^{2} n^{\prime} \mathrm{U}^{\prime} d n^{\prime} d \phi,
$$

and the pressure depends on the term multiplying $w$ contained in $\mathrm{U}^{\text {and }} \mathrm{U}^{\prime}$ or $l^{\prime} u+m^{\prime} v-n^{\prime} w$. If we are only concerned with its main term, that independent of $u v w$, we may write $n^{r}=n$, $p^{\prime}=p$, use statical limits for $n$ and a statical value of $\mathrm{A}^{2}$, and then with $\chi$ for the energy-content of the incident radiation, the pressure is

$$
\frac{\chi}{2 \pi} \cdot \iint\left(1+A^{2}\right) n^{2} d n d \phi . \quad . \quad . \quad .
$$

The terms containing the other section of $\mathrm{U}$, viz. $l u+m v$ will vanish in the integration with regard to $\phi$, if $A^{2}$ is a rational integral function of $(l u+m v)^{2}$ and of $(l v-m i u)$, i.e. of $(u \cos \phi+v \sin \phi)^{2}$ and of $(v \cos \phi-u \sin \phi)$. This is readily seen by taking $v=0$, i. e. by measuring $\phi$ from the component of translation in the reflecting surface, in which case the quantities named vary as $\cos ^{2} \phi$ and $\sin \phi$. The component which must only enter through its square is for each wave-front the component in the reflecting surface along its line of intersection with the wave-front. On the present electromagnetic scheme this will be shown to be true in an exact manner, when $A^{2}$ stands as above for the mean of all polarizations taken for that particular wave-front. Thus there is no work expended in connexion with tangential forces (in the collective result); a further consequence is that in (19) $n w$ may be written for $\mathrm{U}$ and $-n^{\prime} w$ for $\mathrm{U}^{\prime}$, and that all first-order results are independent of $u$ and $v$.

$\S 7$. For perfect reflexion $A^{2}=1$, and the vanishing of the above integrals is obvious, but in that case $u$ and $v$ do not intervene at all, as the variables of the reflected wave are determined independently. of $u$ and $v$. In fact

$$
l: l^{\prime}=m: m^{\prime}=\mathrm{V}-\mathrm{U}: \mathrm{V}-\mathrm{U}^{\prime}
$$

is equivalent to

$$
\text { or to } \quad \sqrt{\sqrt{1-n^{2}}: l^{\prime}=\mathrm{V}-w n: \mathrm{V}+w n^{\prime}}+n^{\prime 2}=\mathrm{V}-w n: \mathrm{V}+w n^{\prime} \text {; }
$$

i. e. $n^{\prime}$ is determined in terms of $n$ precisely as in (4) with only $w$ present. The omission of $u$ and $v$ in the account of perfect reflexion is therefore justified, and that not merely as a preliminary course to secure simplicity in the opening statement. 
In a general formula it may be convenient to measure $n^{\prime}$ in the same direction as $n$; with this arrangement, which may be marked by the use of suffixes, the group of relations for reflexion is

$$
\left.\begin{array}{l}
l_{1}: l_{2}=m_{1}: m_{2}=\mathrm{V}-w n_{1}: \mathrm{V}-w n_{2} \\
=-\left(\mathrm{V} n_{1}-w\right):\left(\mathrm{V} n_{2}-w\right)=\mathrm{V}-\mathrm{U}_{1}: \mathrm{V}-\mathrm{U}_{2}, \\
\text { we may deduce } \\
\quad l_{1} l_{2}+m_{1} m_{2}-n_{1} n_{2}=1-\left(n_{1}+n_{2}\right) w / \mathrm{V},
\end{array}\right\}
$$

and we may deduce

which will be of service later.

Thus

$$
\begin{aligned}
& \frac{1}{l_{1}}\left(l_{1} l_{2}+m_{1} m_{2}-n_{1} n_{2}\right)=-\frac{n_{1} n_{2}}{l_{1}}+\frac{1}{l_{2}}\left(1-n_{2}{ }^{2}\right) \\
& =\frac{1}{l_{2}}-n_{2}\left(\frac{n_{1}}{l_{1}}+\frac{n_{2}}{l_{2}}\right)=\frac{1}{l_{2}}-\frac{w n_{2}}{\mathrm{~V}}\left(\frac{1}{l_{1}}+\frac{1}{l_{2}}\right),
\end{aligned}
$$

using

$$
\begin{array}{r}
l_{2}\left(\mathrm{~V} n_{1}-w\right)+l_{1}\left(\mathrm{~V} n_{2}-w\right)=0, \\
=\frac{1}{l_{1}}\left\{1-\frac{w}{\mathrm{~V}}\left(n_{1}+n_{2}\right)\right\},
\end{array}
$$

using

$$
\frac{1}{l_{2}}\left(1-\frac{w n_{2}}{\mathrm{~V}}\right)=\frac{1}{l_{1}}\left(1-\frac{w n_{1}}{\mathrm{~V}}\right) \text {. }
$$

When $V / \mu$ is written for $V$ and $w / \mu^{2}$ for $w$, the results are applicable to the reflexion of a wave, moving with modified velocity in a dielectric, at a surface sharing the motion of the dielectric. In refruction when $u$ and $v$ exist, $\phi$ always intervenes in the determination of $n^{\prime \prime}$ from $n$, viz., from

follows

$$
l: l^{\prime \prime}=m: m^{\prime \prime}=\mathrm{V}-\mathrm{U}: \frac{\mathrm{V}}{\mu}-\frac{\mathrm{U}^{\prime \prime}}{\mu^{2}}
$$

$$
\frac{1-w n / \nabla}{\sqrt{1-n^{2}}}-\frac{1-w n^{\prime \prime} / \mu V}{\mu \sqrt{1-n^{\prime \prime 2}}}=\left(1-\frac{1}{\mu^{2}}\right)(u \cos \phi+v \sin \phi) / \mathrm{V} .
$$

The first-order relation between ray-cosines may be noticed. We have $l /(1-\mathrm{U} / \mathrm{V})=\mu l^{\prime \prime}\left(1-\mathrm{U}^{\prime \prime} / \mu \mathrm{V}\right)$ exactly, and therefore to the first order

$$
(l-u / V) /(1-\mathrm{U} / \mathrm{V})=\mu\left(l^{\prime \prime}-u / \mu \mathrm{V}\right) /\left(1-\mathrm{U}^{\prime \prime} / \mu \mathrm{V}\right) \text {; }
$$

but to this order

$$
l_{r}=(\mathrm{V} l-u) /(\mathrm{V}-\mathrm{U}) \text { and } l_{r}^{\prime \prime}=\left(\frac{\mathrm{V}}{\mu} l^{\prime \prime}-\frac{u}{\mu^{2}}\right) /\left(\frac{\mathrm{V}}{\mu}-\frac{\mathrm{U}^{\prime \prime}}{\mu^{2}}\right),
$$

and so $l_{r}=\mu l_{r}^{\prime \prime}, m_{r}=\mu m_{r}{ }^{\prime \prime}$.

$\$ 8$. It was noted above, in regard to the formulæ for $\varpi$ and $\varpi^{\prime \prime}$, that the change to a moving standpoint was the 
main fact indicated, and the second and third members of (17) differ only geometrically. When the middle term is used we are in effect interpreting from a moving standpoint radiation which is proceeding in free sether, without reference to reflexion or refraction. If we form a collective relation as before and take first the simpler case $u=v=0$, we have

$$
\begin{aligned}
p^{4} \int(n-w / V)(1-w n / V) d n & =\varpi^{4} \int_{0}^{1} n_{r} d n_{r} /\left\{1-w^{2}\left(1-n_{r}^{2}\right) / V^{2}\right\}^{2} \\
& \text { or } \quad=w^{4} \int_{0}^{1} v d v /\left(1-w^{2} / V^{2}\right) ;
\end{aligned}
$$

the use of the kinematical cosine $\nu=(n-w / V) /(1-w n / V)$ being easier. The result is

$$
p^{4}\left(1-\frac{w}{V}\right)^{3}\left(1+\frac{w}{3 V}\right)=\varpi^{4} /\left(1-\frac{w^{2}}{\nabla^{2}}\right) .
$$

In the general case a double integration is needed, viz. :-

$$
p^{4} \iint\left(n-\frac{w}{\mathrm{~V}}\right)\left(1-\frac{\mathrm{U}}{\mathrm{V}}\right) d n d \phi=w^{4} \iint_{n_{r}} d n_{r} d \phi_{r} /\left[1-\frac{\Sigma u^{2}}{\mathrm{~V}^{2}}+\frac{\mathrm{U}_{r}^{2}}{\mathrm{~V}^{2}}\right]^{2}
$$

but as each of the variables $n_{r} \phi_{r}$ has full range, i.e. 0 to 1 , and 0 to $2 \pi$, the integral on the right is $\pi^{4} /\left(1-\frac{\Sigma u^{2}}{V^{2}}\right)$, that on the left being unchanged. This gives a mean alteration of period due to Doppler's effect and a diminished rate of apprnach of energy. If we are dealing with refraction $\mathrm{B}^{2}$ is used under the sign of integration, because the transformation applies only to a part of the energy, and the integral is more complicated. But the thus obtained is also the $\varpi^{\prime \prime}$ of the refracted ray; there is no further change.

Through the factor belonging to Doppler's effect a proper contribution to mechanical work is levied on the section of the incident energy which suffers refraction. Again, since

$$
p(1-\mathrm{U} / \mathrm{V})=p^{\prime}\left(1-\mathrm{U}^{\prime} / \mathrm{V}\right)=\approx,
$$

the process of reflexion can be divided into two, the first a change from $p$ to $\pi$, the second a change from $\approx$ to $p^{\prime}$, each accompanied by a levy to mechanical work. Thus in effect a levy is made on the whole incident energy, and a further levy on the energy of the reflected wave, and each is associated with a change of standpoint.

The pressure, as pointed out above, can be found (in its main term) by the use of a statical value of $\mathrm{A}^{2}$ and $\mathrm{B}^{2}$; the collective values of $p^{\prime}$ and of and ${ }^{\prime \prime}$ require a knowledge of the way in 
which the components of translation enter into $\mathrm{A}^{2}$ and $\mathrm{B}^{2}$, and on that ground the calculations are deferred to a later stage.

\section{Electromagnetic Scheme.}

In the section of Electromagnetic theory presented here we are concerned almost entirely with a moving standpoint. Such a standpoint seems to be demanded for the consideration of reflexion at a moving surface, and of refraction into a moving dielectric. But it has further applications. When radiation is viewed from a moving standpoint the energy ceases to have purely the character of radiant energy; a part of it assumes a mechanical character. That is, a part of the energy is then radiant energy as interpreted from a moving standpoint, and another part is kinetic energy; and this latter is given by the scalar product of the translation and a momentum belonging to the passage of radiation.

For a dielectric there is a question of the coefficient in the motional term of the energy, and two values claim consideration. One gives a constant velocity of propagation for electromagnetic waves, the other a modified velocity. Both are regarded as actual, the former referring to waves originating in the dielectric, the latter to waves produced by the impact of waves from outside on a moving dielectric. The motion of the dielectric is a motion relative to the standpoint in respect to which the originating wave shows a constant velocity.

The modification is that of Fresnel's formula, and the formula is here exact, not an approximation. This simplifies the character of the equation for transit of energy in reflexion and refraction. Lorentz's equations, when treated exactly, involve a double refraction appertaining to the translation; $i$.e. the propagation of a plane wave is only possible for two modes of polarization, defined by the directions of the translation and of the wave-normal. This introduces great complication into the question of reflexion and refraction, especially as neither velocity of propagation is so easy to deal with as that of Fresnel's formula.

$\$ 9$. For the propagation of electromagnetic waves, the scheme of equations referred to the moving standpoint is taken to be

$$
\overline{\mathrm{V}} \frac{d \mathrm{X}}{d t}=\frac{d \gamma^{\prime}}{d y}-\frac{d \beta^{\prime}}{d z}, \quad \frac{\mathrm{M}}{\mathrm{V}} \frac{d \alpha}{d t}=\frac{d \mathrm{Y}^{\prime}}{d z}-\frac{d \mathrm{Z}^{\prime}}{d y}, \ldots
$$

$$
\mathrm{X}^{\prime}-\mathrm{X}=\epsilon \mathrm{M}(v \gamma-w \beta) / \mathrm{V}, \quad \alpha^{\prime}-\alpha=\epsilon \mathrm{K}(w \mathrm{Y}-v \mathrm{Z}) / \mathrm{V}, \ldots
$$
with $\mathrm{X}^{\prime} \mathrm{Y}^{\prime} \alpha^{\prime} \beta^{\prime}$ continuous at a surface of separation $z=$ constant.

$\mathrm{KX}, \ldots \mathrm{M} \alpha, \ldots$ are the components of electric and magnetic induction, $X^{\prime} \ldots \alpha^{\prime} \ldots$ are the electric and inagnetic 
forces modified by the translation $(u v w)$. The index of refraction will be denoted by $\mu$, i.e. $\mu^{2}=\mathrm{KM}$, the product of the electric and magnetic constants of the medium. The use of the constant $\epsilon$ to cover two cases which are held to be distinct, will be pointed out after a short account of the propagation of a plane wave.

Write

$$
\mathrm{X}=\mathrm{X}_{0} f(l x+m y+n z-\Omega t), \quad \alpha=\alpha_{0} f(l x+m y+n z-\Omega t), \ldots
$$

with the same argument and function throughout; then

$$
\frac{d \mathrm{X}}{d t}=-\Omega \mathrm{X}_{0} f^{\prime}, \text { and } \frac{d \mathrm{X}}{d x}=i \mathrm{X}_{0} f^{\prime}, \ldots
$$

Thus (I.) is replaced by the algebraical equations

$$
\mathrm{K} \Omega \mathrm{X}=\mathrm{V}\left(n \beta^{\prime}-m \gamma^{\prime}\right), \quad \mathrm{M} \Omega \alpha=\mathrm{V}\left(m Z^{\prime}-n \bar{Y}^{\prime}\right) .
$$

From these follow

corresponding to

$$
\Sigma l X=0, \quad \Sigma l a=0 \ldots . .
$$

$$
\Sigma \frac{d \mathrm{X}}{d x}=0, \quad \Sigma \frac{d \alpha}{d x}=0 .
$$

With the help of (II.),

$$
\begin{aligned}
\mathrm{K} \Omega \mathrm{X} & =\mathrm{V}(n \beta-m \gamma)+\epsilon \mathrm{K}\{n(u \mathrm{Z}-v \mathrm{X})-m(v \mathrm{X}-u \mathrm{Y})\} \\
& =\mathrm{V}(n \beta-m \gamma)-\epsilon \mathrm{K} \mathrm{X},
\end{aligned}
$$

writing $\mathrm{U}$ for $\Sigma l u$, and quoting (22). Thus

and

$$
\left.\begin{array}{rl}
\mathrm{KX}(\Omega+\epsilon \mathrm{U}) & =\mathrm{V}(n \beta-m \gamma) ; \\
\mathrm{M} \alpha(\Omega+\epsilon \mathrm{U}) & =\mathrm{V}(m \mathrm{Z}-n \mathrm{Y})
\end{array}\right\} .
$$

follows by similar work. Therefore

$$
\begin{aligned}
\mu^{2} \mathrm{X}(\Omega+\epsilon \mathrm{U})^{2}= & \mathrm{VM}(\Omega+\epsilon \mathrm{U})(n \beta-m \gamma) \\
= & \mathrm{V}^{2}\{n(n \mathrm{X}-l \mathrm{Z})-m(l \mathrm{Y}-m \mathrm{X})\}=\mathrm{V}^{2} \mathrm{X} \\
\text { or } \quad & \quad \Omega+\epsilon \mathrm{U}= \pm \mathrm{V} / \mu . . . . .
\end{aligned}
$$

The two valnes correspond to waves travelling in the sense of the translation and opposite to it, both velocities measured in the sense of the translation : the positive sign will be taken for the standard case.

If we write $x^{\prime}=x+u t, \ldots$ the differential equations (I.) become

where

$$
\frac{\mathrm{K}}{\overline{\mathrm{V}}} \frac{\mathrm{DX}}{\mathrm{D} t}=\frac{d \gamma^{\prime}}{d y^{\prime}}-\frac{d \beta^{\prime}}{d z^{\prime}}, \quad \frac{\mathrm{M}}{\overline{\mathrm{V}}} \frac{\mathrm{D} \alpha}{\mathrm{D} t}=\frac{d \mathrm{Y}^{\prime}}{d z^{\prime}}-\frac{d \mathrm{Z}^{\prime}}{d y^{\prime}}, \ldots
$$

$$
\frac{\mathrm{D}}{\mathrm{D} t}=\frac{d}{d t}+u \frac{d}{d x^{\prime}}+v \frac{d}{d y^{\prime}}+w \frac{d}{d z^{\prime}} \text {. }
$$


The argument is then $l x^{\prime}+m y^{\prime}+n z^{\prime}-(\Omega+\mathrm{U}) t$, and

$$
\Omega+U=\frac{V}{\mu}+U(1-\epsilon) \text {. }
$$

(i.) Thus if $\epsilon=1$, the wave travels with constant velocity $\nabla i \mu$ in relation to $\left(x^{\prime} y^{\prime} z^{\prime}\right)$, and appears to bave the variable velocity $\frac{\mathrm{V}}{\mu}-\mathrm{U}$ in relation to $(x y z)$.

(ii.) But if $\epsilon=\frac{1}{\mu^{2}}$,

$$
\Omega+\mathrm{U}=\frac{\mathrm{V}}{\mu}+\mathrm{U}\left(1-\frac{1}{\mu^{2}}\right), \quad \Omega=\frac{\mathrm{V}}{\mu}-\frac{\mathrm{U}}{\mu^{2}} ;
$$

both are variable unless $\mu=1$, when there is no distinction between (i.) and (ii.).

For a dielectric (i.) is held to apply when the disturbance originates in the dielectric, and the translation in question is a motion relative to the dielectric. Case (ii.) is applicable to waves produced in a dielectric by waves in free wther; the moving standpoint is that of the dielectric itself, and the fixed standpoint is that in respect to which the originating wave travels with constant velocity.

The use of the moving standpoint simplifies the transition from (i.) to (ii.).

$\$ 10$. We proceed to certain relations between the lwo types of variables for a plane wave, and they will be written for case (ii.) for which they are less obvious, because neither stand point shows a contant velocity of propagation. Applying (24) to (23), we have

$$
\text { or } \left.\quad \begin{array}{ll}
\mathrm{KX}=\mu(n \beta-m \gamma), \quad \mathrm{M} \alpha=\mu(m \mathrm{Z}-n \mathrm{Y}) \\
\quad \mu \mathrm{X}=\mathrm{M}(n \beta-m \gamma), \quad \mu \alpha=\mathrm{K}(m \mathrm{Z}-n \mathrm{Y})
\end{array}\right\} ; .
$$

i.e., the relations between the inductions or inducing forces are the same as for the statical case, thongh in (ii.) the velocity is variable. An immediate consequence of (25) is

$$
\mathrm{K} \Sigma \mathrm{X}^{2}=\mathrm{M \Sigma}(n \beta-m \gamma)^{2}=\mathrm{M} \Sigma \alpha^{2}=\frac{\mathrm{K}}{2} \Sigma \mathrm{X}^{2}+\frac{\mathrm{M}}{2} \Sigma \alpha^{2} \equiv \mathrm{E} \text {, }
$$

the last giving a definition of $\mathrm{E}$. We now express $\alpha^{\prime}$ in terms of $a$; thus

$$
\left.\begin{array}{l}
\begin{array}{l}
\alpha^{\prime}=\alpha+\mathrm{K}(w \mathrm{Y}-v \mathrm{Z}) / \mu^{2} \mathrm{~V}=\alpha+\{w(l \gamma-n \alpha)-v(m \alpha-l \beta)\} / \mu \mathrm{V} \\
=\alpha\left(1-\frac{\mathrm{U}}{\mu \mathrm{V}}\right)+\frac{l}{\mu \mathrm{V}} \mathrm{\Sigma}_{u \approx}
\end{array} \\
\text { and similarly } \\
\qquad \mathrm{X}^{\prime}=\mathrm{X}\left(1-\frac{\mathrm{U}}{\mu \mathrm{V}}\right)+\frac{l}{\mu \mathrm{V}} \Sigma_{u} \mathrm{X} .
\end{array}\right\}(27)
$$


Also

$$
\alpha\left(1-\frac{\mathrm{U}}{\mu \mathrm{V}}\right)=\alpha^{\prime}-\frac{l}{\mu \mathrm{V}} \Sigma u \alpha^{\prime}, \text { and } \Sigma \alpha^{\prime}\left(l-\frac{u}{\mu \mathrm{V}}\right)=0 .
$$

with similar properties for $\mathrm{X}$, since $\Sigma u \alpha=\Sigma u \alpha^{\prime}$ and $\Sigma u \mathrm{X}=\Sigma u \mathrm{X}^{\prime}$. Thus the letters with dash denote quantities perpendicular to the ray, not to the wave-normal; also since

$$
\Sigma \alpha^{\prime} \mathbf{X}^{\prime}=\Sigma\left(a^{\prime}-\alpha\right)\left(\mathbf{X}^{\prime}-\mathbf{X}\right)
$$

they are not mutually perpendicular, except for first-order work in (uvw). From (27),

$$
\begin{aligned}
\mathrm{M} \Sigma \alpha \alpha^{\prime} & =\mathrm{M}\left(1-\frac{\mathrm{U}}{\mu \mathrm{V}}\right) \Sigma \alpha^{2}=\mathrm{K}\left(1-\frac{\mathrm{U}}{\mu \mathrm{V}}\right) \Sigma \mathrm{X}^{2}=\mathrm{K}^{\mathrm{N}} \mathrm{XX}^{\prime} \\
& =\frac{\mathrm{K}}{2} \Sigma \mathrm{XX}^{\prime}+\frac{\mathrm{M}}{2} \Sigma \alpha \alpha^{\prime} \equiv \mathrm{S}=\mathrm{E}\left(1-\frac{\mathrm{U}}{\mu \mathrm{V}}\right) ; . \quad .
\end{aligned}
$$

this giving a definition of $\mathrm{S}$. thus

Again we may express $x^{\prime}$ in terms of the electric induction;

$$
\begin{aligned}
\mathrm{V} \alpha^{\prime} & =\mathrm{V} \alpha+\frac{\mathrm{K}}{\mu^{2}}(w \mathrm{Y}-v \mathrm{Z})=\frac{\mathrm{KV}}{\mu}(m \mathrm{Z}-n \mathrm{Y})+\frac{\mathrm{K}}{\mu^{2}}(w \mathrm{Y}-v \mathrm{Z}) \\
& =\mathrm{K}\left\{\left(\frac{\mathrm{V} m}{\mu}-\frac{v}{\mu^{2}}\right) \mathrm{Z}-\left(\frac{\mathrm{V} n}{\mu}-\frac{w}{\mu^{2}}\right) \mathrm{Y}\right\} ;
\end{aligned}
$$

and similarly

$$
\mathrm{VX}^{\prime}=\mathrm{M}\left\{\left(\frac{\mathrm{V} n}{\mu}-\frac{w}{\mu^{2}}\right) \beta-\left(\frac{\mathrm{V} m}{\mu}-\frac{v}{\mu^{2}}\right) \gamma\right\},
$$

relations which involve components of ray-velocity. From (27) and (30),

$$
\begin{aligned}
& \nabla\left(\mathrm{X}^{\prime} \beta^{\prime}-\mathrm{Y}^{\prime} \alpha^{\prime}\right) \\
&= \mathrm{M}\left\{\beta\left(\frac{\mathrm{V} n}{\mu}-\frac{w}{\mu^{2}}\right)-\gamma\left(\frac{\mathrm{V} m}{\mu}-\frac{v}{\mu^{2}}\right)\right\}\left\{\beta\left(1-\frac{\mathrm{U}}{\mu \mathrm{V}}\right)+\frac{m}{\mu \mathrm{V}} \Sigma u \alpha\right\} \\
&-\mathrm{M}\left\{\gamma\left(\frac{\mathrm{V} l}{\mu}-\frac{u}{\mu^{2}}\right)-\alpha\left(\frac{\mathrm{V} n}{\mu}-\frac{w}{\mu^{2}}\right)\right\}\left\{\alpha\left(1-\frac{\mathrm{U}}{\mu \mathrm{V}}\right)+\frac{l}{\mu \mathrm{V}} \Sigma u \alpha\right\} \\
&=\left(\frac{\mathrm{V} n}{\mu}-\frac{w}{\mu^{2}}\right)\left(1-\frac{\mathrm{U}}{\mu \mathrm{V}}\right) \mathrm{M} \Sigma \alpha^{2} \\
&=\left(\frac{\mathrm{V} n}{\mu}-\frac{w}{\mu^{2}}\right)\left(1-\frac{\mathrm{U}}{\mu \mathrm{V}}\right) \mathrm{E}=\left(\frac{\mathrm{V} n}{\mu}-\frac{w}{\mu^{2}}\right) \mathrm{S} . . . . . .
\end{aligned}
$$

From (25),

$$
\mathrm{V}\left(\mathrm{X} \beta-\mathrm{Y}_{\alpha}\right)=\frac{\mathrm{V} n}{\mu} \mathrm{E} . \quad . \quad . \quad . \quad .
$$


This completes the more important algebraical connexions between the two types of variables for a plane wave which are useful for the interpretation of formulæ, and in reflexion and refraction. We may note also that by (II.),

so that

$$
\begin{aligned}
\mathrm{K} \Sigma \mathrm{X}\left(\mathrm{X}^{\prime}-\mathrm{X}\right)= & \mathrm{V}^{-1} \Sigma \mathrm{X}(v \gamma-w \beta) \\
& =\mathrm{V}^{-1} \Sigma \alpha(w \mathrm{Y}-v \mathrm{Z})=\mathrm{M} \Sigma\left(\alpha^{\prime}-\alpha\right) \alpha,
\end{aligned}
$$

$$
\mathrm{K \Sigma XX} \mathrm{X}^{\prime}=\mathrm{M} \Sigma \alpha \alpha^{\prime} \quad \text { whenever } \mathrm{K \Sigma} \mathrm{X}^{2}=\mathrm{M} \Sigma \alpha^{2},
$$

as for plane waves.

$\S 11$. We proceed to the equations for the movement of energy, first within the medium, and then across a surface of separation. We have

$$
\begin{aligned}
\mathrm{K} \Sigma \mathrm{X} \frac{d}{d t}\left(\mathrm{X}^{\prime}-\mathrm{X}\right) & =\mathrm{V}^{-1} \Sigma \mathrm{X}\left(v \frac{d \gamma}{d t}-w \frac{d \beta}{d t}\right) \\
& =\mathrm{V}^{-1} \Sigma \frac{d \alpha}{d t}(w \mathrm{Y}-v \mathrm{Z})=\mathrm{M \Sigma} \Sigma \frac{d a}{d t}\left(\alpha^{\prime}-\alpha\right)
\end{aligned}
$$

and again

$$
\mathrm{M} \Sigma \alpha \frac{d}{d t}\left(\alpha^{\prime}-\alpha\right)=\mathrm{K} \Sigma \frac{d \mathrm{X}}{d t}\left(\mathrm{X}^{\prime}-\mathrm{X}\right)
$$

Hence, by addition,

$$
\mathrm{K} \Sigma \mathrm{X} \frac{d \mathrm{X}^{\prime}}{d t}+\mathrm{M} \Sigma \alpha \frac{d \alpha^{\prime}}{d t}=\mathrm{K} \Sigma \mathrm{X}^{\prime} \frac{d \mathrm{X}}{d t}+\mathrm{M} \Sigma \alpha^{\prime} \frac{d \alpha}{d t},
$$

and therefore

$$
\begin{aligned}
\frac{d}{d t}\left(\frac{\mathrm{K}}{2} \Sigma \mathrm{XX}^{\prime}+\frac{\mathrm{M}}{2} \Sigma \alpha \alpha^{\prime}\right) & =\Sigma \mathrm{K} \frac{d \mathrm{X}}{d t} \mathrm{X}^{\prime}+\Sigma \mathrm{M} \frac{d \alpha}{d t} \alpha^{\prime} \\
& =\mathrm{V} \Sigma\left(\frac{d \gamma^{\prime}}{d y}-\frac{d \beta^{\prime}}{d z}\right) \mathrm{X}^{\prime}+\mathrm{V} \Sigma\left(\frac{d \mathrm{Y}^{\prime}}{d z}-\frac{d \mathrm{Z}^{\prime}}{d y}\right) \alpha^{\prime} \\
& =-\mathrm{V} \Sigma \frac{d}{d z}\left(\mathrm{X}^{\prime} \beta^{\prime}-\mathrm{Y}^{\prime} \alpha\right)
\end{aligned}
$$

or

$$
\frac{d \mathrm{~S}}{d t}+\mathrm{V} \Sigma \frac{d}{d \varepsilon}\left(\mathrm{X}^{\prime} \beta^{\prime}-\mathrm{Y}^{\prime} \alpha^{\prime}\right)=0 ; . . .
$$

i. e., change in $\mathrm{S}$ is due to the flux of which $\mathrm{V}\left(\mathrm{X}^{\prime} \beta^{\prime}-\mathrm{Y}^{\prime} \alpha^{\prime}\right)$ is the $z$-component. For a plane wave this is $\zeta \mathrm{S}$ by (31), $\zeta$ being $z$-component of ray-velocity, and then (33) is expressed by

$$
\frac{d \mathrm{~S}}{d t}+\xi \frac{d \mathrm{~S}}{d x}+\eta \frac{d \mathrm{~S}}{d y}+\zeta \frac{d \mathrm{~S}}{d z}=0 .
$$

The flux across the $z$-face contains precisely those quantities 
$\mathrm{X}^{\prime} \mathrm{Y}^{\prime} \alpha^{\prime} \beta^{\prime}$ which the fundamental equations require to be continuous at a surface of separation. For the transfer of energy in reflexion and refraction something more than the continuity of $\mathrm{V}\left(\mathrm{X}^{\prime} \beta^{\prime}-\mathrm{Y}^{\prime} \alpha^{\prime}\right)$ is wanted, viz. the independence of the flux formulæ for the original and reflected waves expressed by

$$
\mathrm{V}\left(\mathrm{X}^{\prime} \beta^{\prime}-\mathrm{Y}^{\prime} \alpha\right)_{1}+\mathrm{V}\left(\mathrm{X}^{\prime} \beta^{\prime}-\mathrm{Y}^{\prime} \alpha^{\prime}\right)_{2}=\mathrm{V}\left(\mathrm{X}^{\prime} \beta^{\prime}-\mathrm{Y}^{\prime} \alpha^{\prime}\right)_{3} .
$$

the suffixes attaching to the original reflected and refracted waves. If we set out from $\mathrm{X}_{8}{ }^{\prime}=\mathrm{X}_{1}{ }^{\prime}+\mathrm{X}_{2}{ }^{\prime}$, and similar equations for $Y^{\prime} \alpha^{\prime} \beta^{\prime}$, the proof requires

$$
\mathrm{X}_{1}^{\prime} \beta_{2}^{\prime}-\mathrm{Y}_{1}^{\prime} \alpha_{2}^{\prime}+\mathrm{X}_{2}{ }^{\prime} \beta_{1}^{\prime}-\mathrm{Y}_{2}^{\prime} \alpha_{1}^{\prime}=0 \text {, }
$$

or the vanishing of cross-products. Take the first medium for convenience to be æther and apply (27) and (30) exactly as in establishing (31). The quantity which should vanish is

$$
\begin{aligned}
& \Sigma \alpha_{1} \alpha_{2}\left\{\left(\mathrm{~V} n_{1}-w\right)\left(1-\mathrm{U}_{2} / \mathrm{V}\right)+\left(\mathrm{V} n_{2}-w\right)\left(1-\mathrm{U}_{1} / \mathrm{V}\right)\right\} \\
& \quad-\left\{\left(\mathrm{V}-\mathrm{U}_{1}\right) \gamma_{2} \Sigma l_{2} \alpha_{1}+\left(\mathrm{V}-\mathrm{U}_{2}\right) \gamma_{1} \Sigma l_{1} \alpha_{2}\right\} \\
& \quad+\left(1-\Sigma l_{1} l_{2}\right)\left(\gamma_{2} \Sigma u \alpha_{1}+\gamma_{1} \Sigma u \alpha_{2}\right) \\
& \quad+\frac{1}{\mathrm{~V}}\left\{\left(\mathrm{~V} n_{2}-w\right) \Sigma u \alpha_{1} \Sigma l_{1} \alpha_{2}+\left(\mathrm{V} n_{1}-w\right) \Sigma u \alpha_{2} \Sigma l_{2} \alpha_{1}\right\} ;
\end{aligned}
$$

the work so far involving no special relations. The first line vanishes by $(21)$, which also makes

$$
\begin{aligned}
\left(\mathrm{V}-\mathrm{U}_{1}\right) \Sigma l_{2} \alpha_{1}=\left(\mathrm{V}-\mathrm{U}_{2}\right) & \left(l_{1} \alpha_{1}+m_{1} \beta_{1}\right)+\left(\mathrm{V}-\mathrm{U}_{1}\right) n_{2} \gamma_{1} \\
& =\gamma_{1}\left\{\left(\mathrm{~V}-\mathrm{U}_{1}\right) n_{2}-\left(\mathrm{V}-\mathrm{U}_{2}\right) n_{1}\right\},
\end{aligned}
$$

and the second line vanishes when the other part is similarly treated. Again using (21),

and

$$
\left(\mathrm{V} n_{2}-w\right) \Sigma l_{1} \alpha_{2}=\gamma_{2}\left\{n_{1}\left(\mathrm{~V} n_{2}-w\right)+n_{2}\left(\mathrm{~V} n_{1}-w\right)\right\} \text {. }
$$

$$
\begin{aligned}
1-\Sigma l_{1} l_{2} & =-2 n_{1} n_{2}+\frac{w}{\mathrm{~V}}\left(n_{1}+n_{2}\right) \\
& =-\frac{1}{\mathrm{~V}}\left\{n_{1}\left(\mathrm{~V} n_{2}-w\right)+n_{2}\left(\mathrm{~V} n_{1}-w\right)\right\},
\end{aligned}
$$

so that the residue vanishes.

The crass-products do therefore vanish in virtue of $\Sigma l X=0$, $\Sigma l \alpha=0$, and the geometrical laws of reflexion.

Equation (34) written with the formula of interpretation (32) for waves incident in ether on a moving dielectric is

$$
\left(\mathrm{V} n_{1}-w\right) \mathrm{S}_{1}+\left(\mathrm{V} n_{2}-w\right) \mathrm{S}_{2}=\left(\frac{\nabla n_{3}}{\mu}-\frac{w}{\mu^{2}}\right) \mathrm{S}_{8}, .
$$

an equation for the continuity in the rates of arrival and 
departure of $S$ at the surface, in interpreting which we mast remember that $n_{2}$ is measured in the same sense as $n_{1}$, so that for the statical case $n_{2}=-n_{1}$. The moving stand point, that of the diel ctric, is essential for interpretation on the right hand, i.e $\mathbb{S}_{3}$ is to be taken as a substantive quantity. The waves in tree æether should be interpreted with reference to the fixed standpoint for which $\mathrm{E}_{1}$ and $\mathrm{E}_{2}$ are the substantive expressions. Hence, the proper form is

$\left(\mathrm{V} n_{1}-w\right)\left(1-\mathrm{U}_{1} / \mathrm{V}\right) \mathrm{E}_{1}+\left(\mathrm{V} n_{2}-w\right)\left(1-\mathrm{U}_{2} / \mathrm{V}\right) \mathrm{E}_{2}=\left(\frac{\mathrm{V} n_{3}}{\mu}-\frac{w}{\mu^{2}}\right) \mathrm{S}_{3}$

and $\mathrm{E}_{1}$ experiences a transformation at the surface. The interpretation in detail is got by applying

$$
\mathrm{V} n_{1} \mathrm{E}_{1}=\left(\mathrm{V}_{n_{1}}-w\right) \mathrm{S}_{1}+n_{1} \mathrm{U}_{1} \mathrm{E}_{1}+w \mathrm{~S}_{1}
$$

to the two waves in æther, and is:- The rate of progression of energy in theoriginal wave across a fixed plane provides the rate of transit of energy $\left(\frac{V n_{2}}{\mu}-\frac{w}{\mu^{2}}\right) S_{3}$ in the dielectric, the rate of transit of energy $-V n_{2} \mathrm{E}_{2}$ for the reflected wave, the work shown by $n_{1} \mathrm{U}_{1} \mathrm{E}_{1}+n_{2} \mathrm{U}_{2} \mathrm{E}_{2}$, and the energy-content $\mathrm{S}_{1}+\mathrm{S}_{2}$ for the space opened out by the motion. To express the same facts without use of wave-parameters $(n, \mathrm{U})$, (II.) must be applied to the forms for free æther, giving

$$
\left.\begin{array}{rl}
\mathrm{V}(\mathrm{X} \beta-\mathrm{Y} \alpha) & =\mathrm{V}\left(\mathrm{X}^{\prime} \beta^{\prime}-\mathrm{Y}^{\prime} \alpha^{\prime}\right)+w \mathrm{~S}+(w \mathrm{E}-\mathrm{Z \Sigma} u \mathrm{X}-\gamma \mathrm{\Sigma} u \alpha) \\
& =\mathrm{V}\left(\mathrm{X}^{\prime} \beta^{\prime}-\mathrm{Y}^{\prime} \alpha^{\prime}\right)+w \mathrm{~S}+\left(u \mathrm{X}_{z}+v \mathrm{Y}_{z}+w \mathrm{Z}_{z}\right),
\end{array}\right\}
$$

where

$$
\mathrm{X}_{z}=-(\mathrm{XZ}+\alpha \gamma), \quad \mathrm{Z}_{z}=\frac{1}{2}\left(\mathrm{X}^{2}+\mathrm{Y}^{2}-\mathrm{Z}^{2}+\alpha^{2}+\beta^{2}-\gamma^{2}\right),
$$

are the components of a stress.

In (36) the fraction of incident energy which experiences the transformation for reflexion is

$$
\mathrm{A}^{2}=-\frac{\left(\mathrm{V} n_{2}-w\right)\left(1-\mathrm{U}_{2} / \mathrm{V}\right) \mathrm{E}_{2}}{\left(\mathrm{~V} n_{1}-w\right)\left(1-\mathrm{U}_{1} / \mathrm{V}\right) \mathrm{E}_{1}}=\frac{\lambda_{2}{ }^{2} \mathrm{E}_{2}}{\lambda_{1}{ }^{2} \mathrm{E}_{1}}=\frac{p_{2}^{-2} \mathrm{E}_{2}}{p_{1}^{-2} \mathrm{E}_{1}} .
$$

This quantity was required in the previous section, and will be evaluated in solving the problem of refraction. [Or the ratio of the terms in $\mathrm{E}_{1} \mathrm{E}_{2}$ of (36) may be compared directly with the last of (19).]

\$12. We may now consider more fully the expression $\mathrm{S}$,

$$
\begin{aligned}
\mathrm{S} & =\frac{\mathrm{K}}{2} \Sigma X X^{\prime}+\frac{\mathrm{M}}{2} \Sigma \alpha \alpha^{\prime} \\
& =\frac{\mathrm{K}}{2} \Sigma \mathrm{X}^{2}+\frac{\mathrm{M}}{2} \Sigma a^{2}-\frac{\epsilon \mathrm{KM}}{\mathrm{V}} \Sigma u\left(\mathrm{Y}_{\gamma}-\mathrm{Z} \beta\right)
\end{aligned}
$$


The use of $X^{\prime} \ldots \alpha^{\prime} \ldots$ as multipliers in forming the energyequation suggests that in a dynamical scheme they have the character of velocities, confirmed to some extent by their appearance in external conditions; while $\mathrm{KX} . . . \mathrm{M} \alpha .$. have the character of momenta which accords with the use of $\mathrm{K} \frac{d \mathrm{X}}{d t} \ldots$ in the fundamental equations. Thus in $(38),(a)$ is a mixed, and $(b)$ a momental expression for $\mathrm{S}$; and if we start from $(b), \mathrm{X}^{\prime}$ may be defined as

$$
\text { and } \alpha^{\prime} \text { as } \quad \begin{aligned}
\mathrm{X}^{\prime} & \equiv \frac{d \mathrm{~S}}{d(\mathrm{KX})}=\mathrm{X}+\epsilon \mathrm{M}(v \gamma-v \beta) / \mathrm{V}, \\
& \equiv \frac{d \mathrm{~S}}{d(\overline{\mathrm{M} \alpha})}=\alpha+\epsilon \mathrm{K}(w \mathrm{Y}-v \mathrm{Z}) / \mathrm{V},
\end{aligned}
$$

which are the relations II. To express $\mathrm{S}$ in terms of $\mathrm{X}^{\prime} \ldots$ $\alpha^{\prime} \ldots$, the equations II. must be inverted, giving

$$
\begin{aligned}
\mathrm{X}\left(1-\epsilon^{2} \mu^{2} \Sigma u^{2} / \mathrm{V}^{2}\right) & =\mathrm{X}^{\prime}-\epsilon^{2} \mu^{2} \mathrm{~V}-2 u \Sigma u \mathrm{X}^{\prime}-\epsilon \mathrm{MV} V^{-1}\left(v \gamma^{\prime}-w \beta^{\prime}\right) \\
\alpha\left(1-\epsilon^{2} \mu^{2} \Sigma u^{2} / \mathrm{V}^{2}\right) & =\alpha^{\prime}-\epsilon^{2} \mu^{2} \mathrm{~V}^{-2} u \Sigma u \alpha^{\prime}-\epsilon \mathrm{KV} V^{-1}\left(w \mathrm{Y}^{\prime}-v \mathrm{Z}^{\prime}\right) \\
\text { and } & \\
\mathrm{S}^{\prime}\left(1-\epsilon^{2} \mu^{2} \Sigma u^{2} / \mathrm{V}^{2}\right) & =\frac{\mathrm{K}}{2} \Sigma \mathrm{X}^{\prime q}+\frac{\mathrm{M}}{2} \Sigma \alpha^{2}+\epsilon \mu^{2} V^{-1} \Sigma u\left(\mathrm{Y}^{\prime} \gamma^{\prime}-\mathrm{Z}^{\prime} \beta^{\prime}\right) \\
& \quad-\frac{\mathrm{K} \epsilon^{2} \mu^{2}}{2 \mathrm{~V}^{2}}\left(\Sigma u \mathrm{X}^{\prime}\right)^{2}-\frac{\mathrm{M} \epsilon^{2} \mu^{2}}{2 \mathrm{~V}^{2}}\left(\Sigma u \alpha^{\prime}\right)^{2}
\end{aligned}
$$

using $S^{\prime}$ for the expression in terms of letters with dash. The result of differentiating with regard to $\mathrm{X}^{\prime} \ldots \alpha^{\prime} \ldots$ is

$$
\frac{d \mathrm{~S}^{\prime}}{d \overline{\mathrm{X}}^{\prime}}=\mathrm{KX}, \quad \frac{d \mathrm{~S}^{\prime}}{d \alpha^{\prime}}=\mathrm{M} \alpha
$$

as we should expect. It will also be found that

$$
\frac{d \mathrm{~S}^{\prime}}{d w}=\frac{\epsilon \mathrm{KM}}{\mathrm{V}}(\mathrm{X} \beta-\mathrm{Y} \alpha)=-\frac{d \mathrm{~S}}{d w} . . .
$$

The vector of which the $z$-component is $\frac{\mathrm{KM}}{\mathrm{V}}\left(\mathrm{X} \beta-\mathrm{Y}_{\alpha}\right)$ will be denoted by (PQR), and we have for $\epsilon=1$,

$$
\mathrm{S}=\mathrm{E}-\Sigma \mathrm{P} u \text {, or } \mathrm{E}=\mathrm{S}+\Sigma \mathrm{P} u \equiv \mathrm{S}+\mathrm{T} ; .
$$

but for the aberrational case, $\epsilon=1 / \mu^{2}$,

$$
\mathrm{S}=\mathrm{E}-\Sigma \frac{\mathrm{P} u}{\mu^{2}} \text {, or } \mathrm{E}=\mathrm{S}+\Sigma \frac{\mathrm{P} u}{\mu^{2}}=\mathrm{S}+\Sigma \frac{w(\beta \mathrm{X}-\alpha \mathrm{Y})}{\mathrm{V}} \text {. }
$$

Attending first to the normal case (41), $\mathrm{E}$ appears as the 
sum of two terms, the first of which is radiant energy as viewed from a moving standpoint. The second is kinetic in character, i.e. it is a composite term of linetic energy, in which the components of translation are associated with those of a momentum belonging to radiation. If we have an aberrational argument $\Sigma l x-\left(\frac{\mathrm{V}}{\mu}-\frac{\mathrm{U}}{\mu^{2}}\right) t$, and look at the wave from a standpoint in motion $\left(u^{\prime} v^{\prime} w^{\prime}\right)$ relative to the dielectric, i.e. put $x=\xi+u^{\prime} t, \ldots$ the argument is

$$
\Sigma l \xi-\left(\frac{V}{\mu}-\frac{U}{\mu^{2}}-U^{\prime}\right) t .
$$

The corresponding motional term in the energy is

$$
\Sigma \frac{\mathrm{KM}}{\mathrm{V}}\left(\mathrm{X} \beta-\mathrm{Y}_{\alpha}\right)\left(w^{\prime}+\frac{w}{\mu^{2}}\right)
$$

We take the expression for momentum to retain its normal value, while a reduced effective value of the velocity appears in the aberrational case. Thus (40) gives the momentum of radiation in the dielectric when the differentiation is with regard to a velocity relative to the dielectric $\left(w^{\prime}\right)$.

The case as regards aberration is briefly as follows:-For free æther the electric and magnetic sections of energy have expressions $\frac{1}{2} \Sigma X^{2}$ and $\frac{1}{2} \Sigma \alpha^{2}$; when the radiation is viewed from a moving standpoint only a part of this has the character of radiant energy, and there is a kinetic term $\Sigma_{\overline{\mathrm{V}}}^{w}(\mathrm{X} \beta-\mathrm{Y} \alpha)$. In a dielectric $\frac{1}{2} \Sigma X^{2}$ and $\frac{1}{2} \Sigma \alpha^{2}$ are weighted with the coeffcients $K$ and $M$ respectively. Is the kinetic term to be weighted or not? If it is not weighted we have the aberrational effect, i.e. the latter amounts to the retention of the same expression for the part of the formula which denotes kinetic energy. If it is weighted, with a coefficient KM, the meaning corresponds to an alteration of standpoint in viewing a wave which is proceeding with a velocity $\mathrm{V} / \mu$. $\perp \tau$ is here supposed that this case is actual, that a wave originating in a dielectric does travel with constant velocity, and that the translation in the formula is a motion relative to the dielectric.

There is I think a primâ facie case for a real difference between the cases which are here separated; i. e. it is reasonable to expect a difficulty in boarding, or gaining foothold in, the moving medium; and the fact of aberration shows that a wave in such circumstances does not obtain a grip of the full propagating power of the medium.

It is a consequence of this separation of cases that no 
aberrational effect is to be got from a terrestrial source of light without an entry into and passage through a medium in motion relative to the earth.

$\$ 13$. We proceed to the solution of the problem of reflexion and refraction, which will be written for an originating wave in free ather; and for the dielectric $M$ is taken $=1, K=\mu^{2}$, so that the connecting equations (25) and (27) become

$$
\begin{aligned}
& \alpha=\mu(m \mathrm{Z}-n \mathrm{Y}), \quad \mu \mathrm{X}=n \beta-m \gamma \quad . \quad . \quad . \quad . \quad . \quad . \\
& \alpha^{\prime}=\alpha\left(1-\frac{\mathrm{U}}{\mu \mathrm{V}}\right)+\frac{l}{\mu \mathrm{V}} \Sigma u \alpha, \mathrm{X}^{\prime}=\mathrm{X}\left(1-\frac{\mathrm{U}}{\mu \mathrm{V}}\right)+\frac{l}{\mu \mathrm{V}} \Sigma u \mathrm{X} .
\end{aligned}
$$

The solution is simplest when $u=v=0$, so that $\mathrm{U}=n w$, and this case is taken first. For axis of $x$ the line of intersection of wave-front and reflecting surface is taken, and the cases for which the magnetic and electric inductions are along this axis are taken separately.

(i.) Magnetic induction along $x$, i.e. $\beta=\gamma=0$ and $\mathrm{X}=0$. The scheme is, with $r$ for $w / V$,

$$
\begin{aligned}
& \alpha=\mathrm{H}, \quad \mu \mathrm{Y}=-n \mathrm{H}, \quad \mu \mathrm{Z}=m \mathrm{H}, \\
& \left.\alpha^{\prime}=\mathrm{H}\left(1-\frac{r n}{\mu}\right), \beta^{\prime}=0, \mathrm{X}^{\prime}=0, \mathrm{X}^{\prime}=-\frac{\mathrm{H}}{\mu}\left(n-\frac{r}{\mu}\right)\right\} .
\end{aligned}
$$

Writing these for the three waves with $\mathrm{H}, \mathrm{H}^{\prime}$ and $\mathrm{H}^{\prime \prime}$, and equating $\alpha^{\prime} \mathrm{Y}^{\prime}$ at the surface,

$$
\begin{aligned}
& \mathrm{H}(1-r n)+\mathrm{H}^{\prime}\left(1+r n^{\prime}\right)=\mathrm{H}^{\prime \prime}\left(1-\frac{r n^{\prime \prime}}{\mu}\right), \\
& \mathrm{H}(\mathrm{V} n-w)-\mathrm{H}^{\prime}\left(\mathrm{V} n^{\prime}+w_{\rangle}^{\prime}=\mathrm{H}^{\prime \prime}\left(\frac{\mathrm{V} n^{\prime \prime}}{\mu}-\frac{w}{\mu^{2}}\right) ;\right.
\end{aligned}
$$
and

$$
(\mathrm{V} n-w)(1-r n) \mathrm{H}^{2}-\left(\mathrm{V} n^{\prime}+w\right)\left(1+r n^{\prime}\right) \mathrm{H}^{\prime 2}=\left(\frac{\mathrm{V} n^{\prime \prime}}{\mu}-\frac{w}{\mu^{2}}\right)\left(1-\frac{r n^{\prime \prime}}{\mu}\right) \mathrm{H}^{\prime 2}
$$

is the equation for transit of energy, the product $\mathrm{HH}^{\prime}$ vanishing by (21). These equations may be altered in form by introducing the ratios

$$
\lambda: \lambda^{\prime}: \lambda^{\prime \prime}=\mathrm{V}-w n: \mathrm{V}+w n^{\prime}: \frac{\mathrm{V}}{\mu}-\frac{w n^{\prime \prime}}{\mu^{2}},
$$

and by using the kinematical cosines

$$
\frac{n-r}{1-r n}=\nu=\frac{n^{\prime}+r}{1+r n^{\prime}}, \quad \nu^{\prime \prime}=\frac{n^{\prime \prime}-r / \mu}{1-r n^{\prime \prime} / \mu} .
$$


The exact relation between $v$ and $\nu^{\prime \prime}$ is

$$
\left(1-\nu^{2}\right) /\left(1-r^{2}\right)=\mu^{2}\left(1-\nu^{\prime \prime 2}\right) /\left(1-r^{2} / \mu^{2}\right),
$$

and $\nu$ has the range 0 to 1 . The equations are then

$$
\mathrm{H} \lambda+\mathrm{H}^{\prime} \lambda^{\prime}=\mu \mathrm{H}^{\prime \prime} \lambda^{\prime \prime}, \quad\left(\mathrm{H} \lambda-\mathrm{H}^{\prime} \lambda^{\prime}\right) \nu=\mathrm{H}^{\prime \prime} \lambda^{\prime \prime} \nu^{\prime \prime},
$$

the solution is

and

$$
\mathrm{H} \lambda: \mathrm{H}^{\prime} \lambda^{\prime}: \mathrm{H}^{\prime \prime} \lambda^{\prime \prime}=\mu \nu+\nu^{\prime \prime}: \mu \nu-\nu^{\prime \prime}: 2 \nu
$$

$$
\mathrm{A}^{2}=\left(\mathrm{H}^{\prime} \lambda^{\prime}\right)^{2}:(\mathrm{H} \lambda)^{2}=\left(\mu \nu-\nu^{\prime \prime}\right)^{2}:\left(\mu \nu+\nu^{\prime \prime}\right)^{2}
$$

The solution in terms of $n n^{\prime} n^{\prime \prime}$ is of course readily found : the object of using (45) is to present $\mathrm{A}^{3}$ (the fraction determining the partition of energy, $c f . \S 6$ and end of $\S 11$ ) in simple shape.

(ii.) Electric induction along $x$, i.e. $\mathrm{Y}=\mathrm{Z}=0, \alpha=0$. The scheme is

$$
\left.\begin{array}{l}
\beta=n \mathrm{H}, \quad \gamma=-m \mathrm{H}, \quad \mathrm{X}=\mathrm{H} / \mu, \\
\alpha^{\prime}=0, \quad \beta^{\prime}=\mathrm{H}\left(n-\frac{r}{\mu}\right), \quad \mathrm{X}^{\prime}=\frac{\mathrm{H}}{\mu}\left(1-\frac{r n}{\mu}\right), \quad \mathrm{Y}^{\prime}=0
\end{array}\right\}
$$

For continuity we require,

or with $\lambda$,

$$
\begin{aligned}
& \mathrm{H}(1-r n)+\mathrm{H}^{\prime}\left(1+r^{\prime}\right)=\frac{\mathrm{H}^{\prime \prime}}{\mu}\left(1-\frac{r n^{\prime \prime}}{\mu}\right), \\
& \mathrm{H}(\mathrm{V} n-w)-\mathrm{H}^{\prime}\left(\mathrm{V} n^{\prime}+w\right)=\mu \mathrm{H}^{\prime \prime}\left(\frac{\mathrm{V} n^{\prime \prime}}{\mu}-\frac{w}{\mu^{2}}\right)
\end{aligned}
$$

$$
\left.\mathrm{H} \lambda+\mathrm{H}^{\prime} \lambda^{\prime}=\mathrm{H}^{\prime \prime} \lambda^{\prime \prime}, \quad\left(\mathrm{H} \lambda-\mathrm{H}^{\prime} \lambda^{\prime}\right) \nu=\mathrm{H}^{\prime \prime} \lambda^{\prime \prime} \cdot \mu \nu^{\prime \prime}\right\}
$$
and

$\mathrm{H} \lambda: \mathrm{H}^{\prime} \lambda^{\prime}: \mathrm{H}^{\prime \prime} \lambda^{\prime \prime}=\nu+\mu \nu^{\prime \prime}: \nu-\mu \nu^{\prime \prime}: 2 \nu$

$$
A^{2}=\left(\nu-\mu \nu^{\prime \prime}\right)^{2}:\left(\nu+\mu \nu^{\prime \prime}\right)^{2} .
$$

The quantity $\mathrm{A}^{2}$ is invariant for the successive actions which take place when a wave enters a dielectric plate with parallel faces and is repeatedly reflected; for perpendicular incidence it is $(\mu-1)^{2} /(\mu+1)^{2}$.

(iii.) For the general case when $u$ and $v$ exist, the polarizations may be taken in conjunction. Case (i.) is altered to

$$
\begin{aligned}
\alpha^{\prime} & =\mathrm{H}_{1}\left(1-\frac{\mathrm{U}}{\mu \mathrm{V}}\right), & \beta^{\prime} & =\frac{m u \mathrm{H}_{1}}{\mu \mathrm{V}} \\
\mathrm{X}^{\prime} & =0, & \mathrm{VY}^{\prime} & =-\mathrm{H}_{1}\left(\frac{\mathrm{V} n}{\mu}-\frac{w}{\mu^{q}}\right),
\end{aligned}
$$


and case (ii.) to

$$
\begin{aligned}
\alpha^{\prime} & =0, \quad \beta^{\prime}=\mathrm{H}_{2}\left(n-\frac{w}{\mu \mathrm{V}}\right), \\
\mathrm{VX}^{\prime} & =\mathrm{H}_{2}\left(\frac{\mathrm{V} n}{\mu}-\frac{w}{\mu^{2}}\right), \quad \mathrm{VY}^{\prime}=\frac{m u \mathrm{H}_{2}}{\mu^{2}} .
\end{aligned}
$$

The special feature is the appearance of $\beta^{\prime}$ in the first and $\mathrm{Y}^{\prime}$ in the second, in consequence of which a wave with the polarization of (i.) gives rise to a component with the polarization of (ii.), of which the amplitude varies as $u$. The continuity of $\alpha^{\prime} \mathrm{X}^{\prime} \mathrm{Y}^{\prime} \beta^{\prime}$ (in order) in the combined scheme is expressed by

$$
\left.\begin{array}{rl}
\mathrm{H}_{1}(1-\mathrm{U} / \mathrm{V})+\mathrm{H}_{1}\left(1-\mathrm{U}^{\prime} / \mathrm{V}\right) & =\mathrm{H}_{1}^{\prime \prime}\left(1-\mathrm{U}^{\prime \prime} / \mu \mathrm{V}\right), \\
\mathrm{H}_{2}(1-\mathrm{U} / \mathrm{V})+\mathrm{H}_{2}^{\prime}\left(1-\mathrm{U}^{\prime} / \mathrm{V}\right) & =\frac{\mathrm{H}_{2}{ }^{\prime \prime}}{\mu}\left(1-\mathrm{U}^{\prime \prime} / \mu \mathrm{V}\right), \\
\mathrm{H}_{1}\left(n-\frac{w}{\mathrm{~V}}\right)-\mathrm{H}_{1}^{\prime}\left(n^{\prime}+\frac{w}{\mathrm{~V}}\right) & -\frac{m u \mathrm{H}_{2}}{\mathrm{~V}}-\frac{m^{\prime} u \mathrm{H}_{2}^{\prime}}{\mathrm{V}} \\
& =\frac{\mathrm{H}_{1}^{\prime \prime}}{\mu}\left(n^{\prime \prime}-\frac{w}{\mu \mathrm{V}}\right)-\frac{m^{\prime \prime} u \mathrm{H}_{2}^{\prime \prime}}{\mu^{2} \mathrm{~V}}, \\
\mathrm{H}_{2}\left(n-\frac{w}{\mathrm{~V}}\right)-\mathrm{H}_{2}^{\prime}\left(n^{\prime}+\frac{w}{\mathrm{~V}}\right)+ & \frac{m u \mathrm{H}_{1}}{\mathrm{~V}}+\frac{m^{\prime} u \mathrm{H}_{1}^{\prime}}{\mu} \\
& =\mathrm{H}_{2}^{\prime \prime}\left(n^{\prime \prime}-\frac{w}{\mu \mathrm{V}}\right)+\frac{m^{\prime \prime} u \mathrm{H}_{1}^{\prime \prime}}{\mu \mathrm{V}}
\end{array}\right\}
$$

For transit of energy form the product $V\left(\beta^{\prime} X^{\prime}-\alpha^{\prime} Y^{\prime}\right)$, and the result is

$$
\begin{array}{r}
(\mathrm{V} n-w)(1-\mathrm{U} / \mathrm{V})\left(\mathrm{H}_{1}{ }^{2}+\mathrm{H}_{2}{ }^{2}\right)-\left(\nabla n^{\prime}+w\right)\left(1-\mathrm{U}^{\prime} / \mathrm{V}\right)\left(\mathrm{H}_{1}{ }^{\prime 2}+\mathrm{H}_{2}{ }^{\prime 2}\right) \\
=\left(\frac{\mathrm{V}_{n}^{\prime \prime}}{\mu}-\frac{w}{\mu^{2}}\right)\left(1-\frac{\mathrm{U}^{\prime \prime}}{\mu \mathrm{V}}\right)\left(\mathrm{H}_{1}{ }^{\prime \prime 2}+\mathrm{H}_{2}{ }^{\prime 2}\right),
\end{array}
$$

all products disappearing in virtue of the geometrical equations of reflexion.

With the object of getting at the ratios $\mathrm{H} \lambda: \mathrm{H}^{\prime} \lambda^{\prime}: \mathrm{H}^{\prime \prime} \lambda^{\prime \prime}$ and at the value of $A^{2}, \lambda$ is introduced as before with

also we write

$$
\lambda: \lambda^{\prime}: \lambda^{\prime \prime}=m: m^{\prime}: m^{\prime \prime}=\mathrm{V}-\mathrm{U}: \mathrm{V}-\mathrm{U}^{\prime}: \frac{\mathrm{V}}{\mu}-\frac{\mathrm{U}^{\prime \prime}}{\mu^{2}} ;
$$

$$
\mathrm{N}=(\mathrm{V} n-w) /(\mathrm{V}-\mathrm{U}), \quad \mathrm{N}^{\prime \prime}=\left(\frac{\mathrm{V}_{n}{ }^{\prime}}{\mu}-\frac{w}{\mu^{2}}\right) /\left(\frac{\mathrm{V}}{\mu}-\frac{\mathrm{U}^{\prime \prime}}{\mu^{2}}\right),
$$

$\mathrm{M}=m /(1-\mathrm{U} / \mathrm{V})$, with $\mathrm{N}^{\prime}=\mathrm{N}$ and $\mathrm{M}^{\prime}=\mathrm{M}$, and $k$ for $1-\frac{1}{\mu^{2}}$

Phil. Mrag. S. 6. Vol. 9. No. 51. March $1905 . \quad$ Z 
The scheme is then

$$
\left.\begin{array}{c}
\mathrm{H}_{1} \lambda+\mathrm{H}_{1}{ }^{\prime} \lambda^{\prime}=\mu \mathrm{H}_{1}{ }^{\prime \prime} \lambda^{\prime \prime}, \quad \mathrm{H}_{2} \lambda+\mathrm{H}_{2}{ }^{\prime} \lambda^{\prime}=\mathrm{H}_{2}{ }^{\prime \prime} \lambda^{\prime \prime}, \\
\left(\mathrm{H}_{1} \lambda-\mathrm{H}_{1}{ }^{\prime} \lambda^{\prime}\right) \mathrm{N}=\mathrm{H}_{1}{ }^{\prime \prime} \lambda^{\prime \prime} \mathrm{N}^{\prime \prime}+k u \mathrm{MH}_{2}{ }^{\prime \prime} \lambda^{\prime \prime}, \\
\left(\mathrm{H}_{2} \lambda-\mathrm{H}_{2}{ }^{\prime} \lambda^{\prime}\right) \mathrm{N}=\mathrm{H}_{2}{ }^{\prime \prime} \lambda^{\prime \prime} \mu \mathrm{N}^{\prime \prime}-\mu k u \mathrm{MH}_{1}{ }^{\prime \prime} \lambda^{\prime \prime},
\end{array}\right\}
$$

and the solution is

$$
\left.\begin{array}{l}
\mathrm{H}_{1}{ }^{\prime} \lambda^{\prime}\left\{\overline{\mathrm{N}+\mu \mathrm{N}^{\prime \prime}} \overline{\mu \mathrm{N}+\mathrm{N}^{\prime \prime}}+\mu k^{2} u^{2} \mathrm{M}^{2} / \mathrm{V}^{2}\right\} \\
\quad=\mathrm{H}_{1} \lambda\left\{\overline{\mu \overline{\mathrm{N}-\mathrm{N}^{\prime \prime}}} \overline{\mathrm{N}+\mu \mathrm{N}^{\prime \prime}}-\mu k^{2} u^{2} \mathrm{M}^{2} / \mathrm{V}^{2}\right\}-\frac{2 \mu k u \mathrm{MN}}{\mathrm{V}} \mathrm{H}_{2} \lambda, \\
\left.\mathrm{H}_{2} \lambda^{\prime} \overline{\left\{\overline{\mathrm{N}+\mu \mathrm{N}^{\prime \prime}}\right.} \overline{\mu \mathrm{N}+\mathrm{N}^{\prime \prime}}+\mu k^{2} u^{2} \mathrm{M}^{2} / \mathrm{V}^{2}\right\} \\
\left.\quad=\mathrm{H}_{2} \lambda \overline{\mathrm{N}-\mu \mathrm{N}^{\prime \prime}} \overline{\mu \mathrm{N}+\mathrm{N}^{\prime \prime}}-\mu k^{2} u^{2} \mathrm{M}^{2} / \mathrm{V}^{2}\right\}+\frac{2 \mu k u \mathrm{MN}}{\mathrm{V}} \mathrm{H}_{i} \lambda .
\end{array}\right\}
$$

If $u=0$, we note that the cases are separated, and the solutions differ from (i.), (ii.) in having $\mathrm{N}, \mathrm{N}^{\prime \prime}$ for $v, v^{\prime \prime}$. The general value of $A^{2}$ is

$$
\left(\mathrm{H}_{1}^{\prime 2}+\mathrm{H}_{2}^{\prime 2}\right) \lambda^{\prime 2}:\left(\mathrm{H}_{1}^{2}+\mathrm{H}_{2}^{2}\right) \lambda^{2} \text {. }
$$

Its dependence on the original polarization is shown by writing

when $A^{2}$ has the form

$$
\mathrm{H}_{1}=\mathrm{H} \cos \psi, \quad \mathrm{H}_{2}=\mathrm{H} \sin \psi,
$$

$$
\alpha \cos ^{2} \psi+\beta \sin ^{2} \psi+2 \gamma \sin \psi \cos \psi \text {. }
$$

Here $\alpha$ and $\beta$ contain $u^{2}$ explicitly, while $\gamma$ shows the first power of $u$. When the mean of $A^{2}$ for all orientations with a given wave-front is taken, the term in $\gamma$ vanishes and the mean is $\frac{1}{2}(\alpha+\beta)$, which contains $u^{2}$ explicitly. Now $\mathrm{N} \mathrm{N}^{\prime \prime} M$ contain $v$ implicitly, bat not $u$, since $l=0$ in $U=l u+m v+n w$. Thus the component of translation which only appears through its square is that along the line of intersection of the wavefront and the reflecting surface. This is the condition stated at the end of $\S 6$, that the tangential forces shall do no work in the aggregate, and it also secures a simplification of the general equations for the collective relations.

$\S 14$. In finding the pressure and the relations between $p p^{\prime}$ and $\varpi$ we shall be content with first-order work; and as the influence of $u$ and $v$ only appears in second-order terms, we shall suppose that $w$ only exists. The use of the kinematical cosine $\nu$ is more convenient than $n$ because $\mathrm{A}^{2}$ is expressed in terms of $\nu v^{\prime \prime}$, and $v$ has the range 0 to 1 , while to the first order $1-\nu^{2}=\mu^{2}\left(1-\nu^{\prime / 2}\right)$. The relations are

$$
\begin{aligned}
& p^{4} \int \mathrm{A}^{2}(n-r)(1-r n) d n=p^{4} \int \mathrm{A}^{2}\left(n^{\prime}+r\right)\left(1+r n^{\prime}\right) d n^{\prime}, \\
& p^{4} \int \mathrm{B}^{2}(n-r)(1-r n) d n=\sigma^{4} \int \mathrm{B}^{2} \nu d \nu .
\end{aligned}
$$


and Electromagnetic Theory.

Since $n-r=v(1-r n)$, and $d v /\left(1-r^{2}\right)=d n /(1-r n)^{2}$, $(n-r)(1-r n) d n=\nu d v(1-r n)^{4} /\left(1-r^{2}\right)=\nu d v(1-4 r v)$ to the order required. Thus

and

$$
p^{4} \int_{0}^{1} \mathrm{~A}^{2} v d v(1-4 r v)=p^{\prime 4} \int_{0}^{1} \mathrm{~A}^{2}(1+4 r v) v d v
$$

$$
p^{4} \int_{0}^{1} \mathrm{~B}^{2} v d v(1-4 r v)=\sigma^{4} \int_{0}^{1} \mathrm{~B}^{2} v d v
$$

Since $A^{2}+B^{2}=1$, only two independent integrals are wanted for each type of polarization, viz. $\int B^{2} v d v$ and $\int \mathrm{B}^{2} v^{2} d v$, say $\mathrm{B}_{1}$ and $\mathrm{B}_{2}$. Then

In terms of these

$$
A_{1}+B_{1}=\frac{1}{2} \text { and } A_{2}+B_{2}=\frac{1}{3} \text {. }
$$

$$
p^{\prime}=p\left(1-2 r \mathrm{~A}_{2} / \mathrm{A}_{1}\right) \text { and } \sigma=p\left(1-r \mathrm{~B}_{2} / \mathrm{B}_{1}\right) ;
$$

and the pressure is

$$
\chi \int \nu^{2} d v\left(1+\mathrm{A}^{2}\right)=\chi\left(\frac{1}{3}+\mathrm{A}_{2}\right)=\chi\left(\frac{2}{3}-\mathrm{B}_{2}\right) .
$$

Also each of these integrals is a mean of that for the polarizations (i.) and (ii.) above, say $2 \mathrm{~B}_{1}=\mathrm{B}_{1}{ }^{\prime}+\mathrm{B}_{1}{ }^{\prime \prime}$. We may now replace $v v^{\prime \prime}$ in these formulæ by the statical $n n^{\prime \prime}$.

(i.) With $\left.\mathrm{B}^{2}=4 \mu n n^{\prime \prime}\right]\left(n+\mu n^{\prime \prime}\right)^{2}, \mu n^{\prime \prime}=\sqrt{n^{2}+\mu^{2}-1}$,

and

$$
\frac{1}{n+\mu n^{\prime}}=\frac{\sqrt{n^{4}+\mu^{2}-1}-n}{\mu^{2}-1},
$$

$$
\begin{aligned}
\frac{\left(\mu^{2}-1\right)^{2}}{4} \mathrm{~B}_{1}{ }^{\prime}= & \int_{0}^{1} n^{2} d n \sqrt{n^{2}+\mu^{2}-1}\left(\sqrt{n^{2}+\mu^{2}-1}-n\right)^{2} \\
& =\int_{0}^{1} d n\left\{n^{2}\left(2 n^{2}+\mu^{2}-1\right) \sqrt{n^{2}+\mu^{2}-1}-2 n^{3}\left(n^{2}+\mu^{2}-1\right)\right. \\
= & {\left[\frac{n}{3}\left\{\left(n^{2}+\mu^{2}-1\right)^{\frac{6}{2}}-\left(\mu^{2}-1\right)\left(n^{2}+\mu^{2}-1\right)^{\frac{3}{2}}\right\}-\frac{\mu^{2}-1}{2} n^{4}-\frac{n^{6}}{3}\right]_{0}^{1} } \\
= & \frac{\mu^{3}-1}{3}-\frac{\mu^{2}-1}{2} ;
\end{aligned}
$$

$$
\begin{aligned}
\frac{\left(\mu^{2}-1\right)^{2} \mathrm{~B}_{2}{ }^{\prime}=}{4}=\int_{0}^{1} d n\left\{n^{3}\left(2 n^{2}+\mu^{2}-1\right) \sqrt{n^{2}+\mu^{2}-1}-2 n^{4}\left(n^{2}+\mu^{2}-1\right)\right\} \\
=\left[\frac{2}{7}\left(n^{2}+\mu^{2}-1\right)^{\frac{7}{2}}-\frac{3\left(\mu^{2}-1\right)}{5}\left(n^{2}+\mu^{2}-1\right)^{\frac{5}{2}}\right. \\
\left.\quad \quad \quad+\frac{\left(\mu^{2}-1\right)^{2}}{3}\left(n^{2}+\mu^{2}-1\right)^{\frac{3}{2}}-\frac{2 n^{7}}{7}-\frac{2 n^{5}\left(\mu^{2}-1\right)}{5}\right]_{0}^{1} \\
=\frac{2 \mu^{7}}{105}-\frac{\mu^{5}}{15}+\frac{\mu^{3}}{3}-\frac{2}{105}\left(\mu^{2}-1\right)^{\frac{7}{2}}-\frac{2}{7}-\frac{2}{5}\left(\mu^{2}-1\right) .
\end{aligned}
$$


340

(ii.) For $\mathrm{B}^{2}=4 \mu n n^{\prime \prime} /\left(\mu n+n^{\prime \prime}\right)^{2}$, the work is more troublesome.

Put $2 n y=\sqrt{\mu^{2}-1}\left(1-y^{2}\right), \quad 2 \mu n^{\prime \prime} y=\sqrt{\mu^{2}-1}\left(1+y^{2}\right)$, and write

then

$$
c^{2}=\frac{\mu^{2}+1}{\mu^{2}-1}, \quad y_{0}^{2}=\frac{\mu-1}{\mu+1} ;
$$

$$
\begin{aligned}
\mathbf{B}_{1}^{\prime \prime} & =\int_{0}^{1} \frac{4 \mu n^{2} n^{\prime \prime} d n}{\left(\mu n+n^{\prime \prime}\right)^{2}}=\frac{\mu^{2}}{\mu^{2}-1} \int_{y_{0}}^{1} \frac{\left(1-y^{4}\right)^{2} d y}{y^{3}\left(c^{2}-y^{2}\right)^{2}} \\
& =\frac{\mu^{2}}{\mu^{2}-1} \int_{y_{0}}^{1} d y\left\{y+\frac{2}{c^{6} y}+\frac{1}{c^{4} y^{3}}-\frac{2\left(c^{8}-1\right) y}{c^{6}\left(c^{2}-y^{2}\right)}+\frac{\left(c^{4}-1\right)^{2} y}{c^{4}\left(c^{3}-y^{2}\right)^{2}}\right\} \\
& =\left[\frac{y^{2}}{2}+\frac{2}{c^{6}} \log y-\frac{1}{2 c^{4} y^{2}}+\frac{c^{8}-1}{c^{6}} \log \left(c^{2}-y^{2}\right)+\frac{\left(c^{4}-1\right)^{2}}{2 c^{4}\left(c^{2}-y^{2}\right)}\right]_{y_{0}}^{1} \\
& =\frac{\mu^{2}}{(\mu+1)^{2}(\mu-1)}+\frac{\mu^{2}(\mu+1)}{\left(\mu^{2}+1\right)^{2}}+\frac{4 \mu^{5}}{(\mu-1)(\mu+1)^{2}\left(\mu^{2}+1\right)^{2}} \\
& \quad+\frac{\mu^{2}\left(\mu^{2}-1\right)^{2}}{\left(\mu^{2}+1\right)^{3}} \log \frac{\mu+1}{\mu-1}-\frac{8 \mu^{4}\left(\mu^{4}+1\right)}{\left(\mu^{2}-1\right)^{2}\left(\mu^{2}+1\right)^{3}} \log \mu . \\
& \mathbf{B}_{2}^{\prime \prime}=\frac{\mu^{2}}{2 \sqrt{\mu^{2}-1}} \int_{y_{0}}^{1} \frac{\left(1-y^{2}\right)^{3}\left(1+y^{2}\right)^{2} d y}{y^{4}\left(c^{2}-y^{2}\right)^{2}}=\frac{\mu^{2}}{2 \sqrt{\mu^{2}-1}} \int_{y_{0}}^{1} f^{\prime}(y) d y,
\end{aligned}
$$

where

$$
\begin{aligned}
f^{\prime}(y)= & -y^{2}-\left(2 c^{2}-1\right)-\frac{c^{2}-2}{c^{6} y^{2}}+\frac{1}{c^{4} y^{4}} \\
& +\frac{3 c^{4}-2 c^{2}-2-c^{-4}+2 c^{-6}}{c^{2}-y^{2}}-\frac{\left(c^{4}-1\right)^{2}\left(c^{2}-1\right)}{c^{4}\left(c^{2}-y^{2}\right)^{2}} \\
f(y)= & -\frac{y^{3}}{3}-\left(2 c^{2}-1\right) y+\frac{c^{2}-2}{c^{6} y}-\frac{1}{3 c^{4} y^{3}} \\
& +\frac{\left(c^{2}+1\right)\left(c^{2}-1\right)^{2}\left(5 c^{4}+2 c^{2}+5\right)}{4 c^{7}} \log \frac{c+y}{c-y}-\frac{\left(c^{4}-1\right) 2\left(c^{2}-1\right)}{2 c^{6}} \frac{y}{c^{2}-y^{2}}
\end{aligned}
$$

giving

$$
\begin{aligned}
\mathrm{B}_{2}{ }^{\prime \prime} & =\frac{\mu^{2}}{2(\mu+1)}\left[\frac{\mu-1}{3(\mu+1)}+\frac{\mu^{2}+3}{\mu^{2}-1}+\frac{(\mu+1)^{3}(\mu-1)\left(\mu^{2}-3\right)}{\left(\mu^{2}+1\right)^{3}}\right. \\
& \left.+\frac{(\mu+1)^{4}}{3\left(\mu^{2}+1\right)^{2}}+\frac{8 \mu^{3}}{\left(\mu^{2}+1\right)^{3}\left(\mu^{2}-1\right)}\right]-\frac{4 \mu^{2}\left(\mu^{8}+12 \mu^{4}+2\right)}{3\left(\mu^{2}+1\right)^{3}\left(\mu^{2}-1\right)^{\frac{3}{2}}} \\
& +\frac{4 \mu^{4}\left(3 \mu^{2}+2\right)}{\left(\mu^{2}+1\right)^{\frac{7}{2}}\left(\mu^{2}-1\right)^{2}} \log \left[\mu\left(\mu^{2}+\sqrt{\mu^{4}-1}\right) /\left\{\mu^{2}-\mu+1+(\mu-1) \sqrt{\mu^{2}+1}\right\}\right] .
\end{aligned}
$$


and Electromagnetic Theory.

When $\mu$ is little greater than 1 , each polarization gives approximately

$$
\begin{array}{rlrl} 
& \mathrm{B}_{1}=\frac{1}{2}-\frac{\mu^{2}-1}{12}, & \mathrm{~B}_{2}=\frac{1}{3}-\frac{8}{105}\left(\mu^{2}-1\right)^{\frac{3}{2}}, \\
\text { i. e. } \quad \mathrm{A}_{1}=\frac{\mu^{2}-1}{12}, & \mathrm{~A}_{2}=\frac{8}{105}\left(\mu^{2}-1\right)^{\frac{3}{2}} .
\end{array}
$$

Hence the values of the ratios

$$
B_{2}: B_{1}=\frac{2}{3}, \quad \text { and } A_{2}: A_{1}=\frac{32}{35} \sqrt{\mu^{2}-1} \text {. }
$$

When $\mu$ is indefinitely great,

$$
\mathrm{B}_{1}^{\prime}=\frac{4}{3 \mu}, \quad \mathrm{B}_{2}^{\prime}=\frac{1}{\mu}, \quad \mathrm{B}_{1}^{\prime \prime}=\frac{4}{\mu}, \quad \mathrm{B}_{2}^{\prime \prime}=\frac{2}{\mu} ;
$$

and for the means

$$
\mathrm{B}_{1}=\frac{8}{3 \mu}, \quad \mathrm{B}_{2}=\frac{3}{2 \mu} .
$$

Since $A_{1}+B_{1}=\frac{1}{2}, A_{2}+B_{2}=\frac{1}{3}$, the ratios are

$$
B_{2}: B_{1}=\frac{9}{16} \text { and } A_{2}: A_{1}=\frac{2}{3} \text {. }
$$

The formula for pressure $\chi\left(\frac{2}{3}-B_{3}\right)$ gives for $\mu$ little greater than 1 . the value

$$
\chi\left\{\frac{1}{3}+\frac{8}{105}\left(\mu^{2}-1\right)^{\frac{3}{2}} \ldots\right\}
$$

and for $\mu$ very great

$$
\chi\left\{\frac{2}{3}-\frac{3}{2 \mu} \ldots\right\} \text {. }
$$

The notion of interpreting the two sides of (14) in a similar way so as to count a term $-\frac{V \lambda^{\prime \prime-4}}{\mu^{3}} n^{\prime \prime} U^{\prime \prime} d n^{\prime \prime} d \phi$ with the terms of the other side in the mechanical rate of working was rejected, $c f . \S 5$. The consequences of such an interpretation may be noted. The main term in pressure when statical values are introduced, as for $(20)$, is then

$$
\frac{x}{2 \pi} \iint\left\{n\left(1+\mathrm{A}^{2}\right)-n^{\prime \prime} \mathrm{B}^{2} \psi \mu\right\} n d n d \phi
$$

and the new term means a back pressure from the dielectric 
on the rther. The expression in brackets is for one principal polarization always negative for some part of the range of $n$; for the other principal polarization this is true if $\mu^{2}<2$. The integral for either or for a mean of both is positive in the upper range of $\mu$, and when $\mu$ is infinite has the limit which belongs to perfect reflexion; but it is negative for a finite though short range of $\mu$ starting from $\mu=1$. It seems difficult to accept an interpretation which gives a back pressure from the dielectric on the æther, or involves the attraction of a dielectric by impinging waves, if $\mu$ has a value below a certain limit (different for the two polarizations if taken separately), and for higher values a repulsive action. [Of course entry into an infinite dielectric is in question, not the resultant of actions on entry and emergence.]

$\S 15$. In the original argument it appeared that the result of differentiating the equations giving the laws of reflexion and refraction, was a connexion between cubes of periods, which was then altered by the use of the factors

$$
p(1-\mathrm{U} / \mathrm{V})=p^{\prime}\left(1-\mathrm{U}^{\prime} / \mathrm{V}\right)=\varpi ;
$$

and the possibility of using

$$
f\{p(1-\mathrm{U} / \mathrm{V})\}=f\left\{p^{\prime}\left(1-\mathrm{U}^{\prime} / \mathrm{V}\right)\right\}=f(\varpi)
$$

in place of the linear factor was just mentioned, of. $\S 3$. We now examine the consequences of supposing this done, the method remaining in other respects as before. The relations between $p, p^{\prime}$, and $\varpi$, when only $w$ exists, then become

$$
\left.\begin{array}{l}
p^{3} \int_{0}^{1} \mathrm{~A}^{2} v d \nu(1-3 r v) f\{p(1-r v)\}=p^{3} \int_{0}^{1} \mathrm{~A}^{2} v d \nu(1+3 r v) f\{p(1+r v)\}, \\
p^{3} \int_{0}^{1} \mathrm{~B}^{2} \nu d v(1-3 r v) f\{p(1-r v)\}=\varpi^{8} f^{\prime}(\varpi) \int_{0}^{1} \mathrm{~B}^{2} \nu d v .
\end{array}\right\}
$$

With first-order work $f\{p(1-r v)\}=f(p)-p r v f^{\prime}(p)$; i. e.

$$
\begin{gathered}
p^{3} \int_{0}^{1} \mathrm{~A}^{2} v d v\left\{f(p)-r v\left(3 f(p)+p f^{\prime}(p)\right)\right\}=p^{3} \int_{0}^{1} \mathrm{~A}^{2} v d \nu\left\{f\left(p^{\prime}\right)+r v\left(3 f\left(p^{\prime}\right)+p^{\prime} f^{\prime}\left(p^{\prime}\right)\right.\right. \\
\text { or } \\
\mathrm{A}_{1} p^{3} f(p)-r \mathrm{~A}_{2}\left(3 p^{3} f(p)+p^{4} f^{\prime}(p)\right)=\mathrm{A}_{1} p^{\prime 3} f\left(p^{\prime}\right)+r \mathrm{~A}_{2}\left(3 p^{\prime 3} f\left(p^{\prime}\right)+p^{\prime 4} f^{\prime}\left(p^{\prime}\right)\right) \\
\text { If now } p^{\prime}=p\left(1+\epsilon^{\prime}\right) \\
p^{\prime 3} f\left(p^{\prime}\right)=p^{8} f(p)+\epsilon^{\prime}\left(3 p^{3} f(p)+p^{4} f^{\prime}(p)\right)
\end{gathered}
$$

and therefore

$$
\left(3 p^{3} f(p)+p^{4} f^{\prime}(p)\right)\left(2 r \mathrm{~A}_{2}+\epsilon^{\prime} \mathrm{A}_{1}\right)=0
$$


i. e. $\epsilon^{\prime}=-2 r \mathrm{~A}_{2} / \mathrm{A}_{1}$, whatever the function is, because the apparent case of exception $3 p^{3} f(p)+p^{4} f^{\prime}(p)=0$ is one which makes the transformation nugatory. In a similar way if

$$
\varpi=p\left(1+\epsilon^{\prime \prime}\right) \text {, we get } \epsilon^{\prime \prime}=-r \mathrm{~B}_{2} / \mathrm{B}_{1} \text {. }
$$

Again, if this function is used the estimate of the rate of conversion to mechanical work at the surface, instead of depending on $r n$ in the factor $1-r n$, must depend on the first-order term in $f\{p(1-r n)\}$. The main term in pressure would then be proportional to

$$
p^{4} f^{\prime}(p) \int n^{2}\left(1+\mathrm{A}^{2}\right) d n ;
$$

or, if we were dealing with $p^{2} f(p) d p$ instead of $p^{3} f(p)$, it would be proportional to

$$
p^{3} f^{\prime}(p) d p \int n^{2}\left(1+A^{2}\right) d n .
$$

Thus on the geometrical side we should have to deal with the same fraction $\frac{1}{3}+A_{2}$ or $\frac{2}{3}-B_{2}$; but this would be a fraction of $p^{3} f^{\prime}(p) d p$, the energy varying as $p^{2} f(p) d p$; or the whole ratio of pressure to the energy-content of the incident wave would be

$$
\frac{p f^{\prime}(p)}{f(p)}\left(\frac{2}{3}-B_{2}\right)
$$

When the fourth power is used

$$
f(p)=p, \text { or } p f^{\prime}(p): f(p)=1 ;
$$

but in any other case the energy converted would be a different fraction of the energy-content in different parts of the periodic scale. Thus, so far as first-order work is concerned, when $w / \mathrm{V}$ is small, the choice of a different function does not alter the collective relations; but it does alter the way in which pressure is related to energy-content, $i$ e. it implies a difference of efficiency as regards the production of mechanical work by pressure, depending on the period of vibration.

$\$ 16$. There are some quantities continuous at a surface of separation which have not received attention.

(i.) $\mathrm{KZ}$ and $\mathrm{M} \gamma$ are continuous; $i$. $e$, for reflexion and refraction $Z_{1}+Z_{2}=K Z_{3}$. The continuity follows from the differential equations; but in view of any doubt possibly arising in connexion with the forms $\frac{d}{d t}$ and $\frac{\mathrm{D}}{\mathrm{D} t}$, an independent proof for the reflexion of a wave is given. It may be remarked 
in this connexion that (I) is always to be regarded as the fundamental form of the equations.

$$
\begin{aligned}
\mathrm{KZ}_{3}= & \mu\left(m_{3} \alpha_{3}-l_{3} \beta_{3}\right)=\mu\left(m_{3} \alpha_{3}{ }^{\prime}-l_{3} \beta_{3}{ }^{\prime}\right) /\left(1-\mathrm{U}_{3} / \mu \mathrm{V}\right), \\
& \text { since } \quad a_{3}{ }^{\prime}=\alpha_{3}\left(1-\frac{\mathrm{U}_{3}}{\mu \mathrm{V}}\right)+\frac{l_{3}}{\mu \mathrm{V}} \Sigma u \alpha_{3}, \\
= & \mu\left\{m_{3}\left(\alpha_{1}{ }^{\prime}+\alpha_{2}{ }^{\prime}\right)-l_{3}\left(\beta_{1}{ }^{\prime}+\beta_{2}{ }^{\prime}\right)\right\} /\left(1-\mathrm{U}_{3} / \mu \mathrm{V}\right) \\
= & \left(m_{1} \alpha_{1}{ }^{\prime}-l_{1} \beta_{1}\right) /\left(1-\mathrm{U}_{1} / \mathrm{V}\right)+\left(m_{2} \alpha_{2}{ }^{\prime}-l_{2} \beta_{2}{ }^{\prime}\right) /\left(1-\mathrm{U}_{2} / \mathrm{V}\right), \\
& \text { since } \quad \frac{m_{1}}{1-\mathrm{U}_{1} / \mathrm{V}}=\frac{m_{2}}{1-\mathrm{U}_{2} / \mathrm{V}}=\frac{\mu m_{3}}{1-\mathrm{U}_{3} / \mu \mathrm{V}} \\
= & m_{1} \alpha_{1}-l_{1} \beta_{1}+m_{2} \alpha_{2}-l_{2} \beta_{2}=\mathrm{Z}_{1}+\mathrm{Z}_{2} .
\end{aligned}
$$

(ii.) $\mathrm{KZ}, \mathrm{M}_{\gamma}$ being continuous as well as $\alpha^{\prime} \beta^{\prime} \mathrm{X}^{\prime} \mathrm{Y}^{\prime}$, we expect $\mathrm{KZX}^{\prime}+\mathrm{M}_{\gamma \alpha^{\prime}}$, and $\mathrm{KZY^{ \prime }}+\mathrm{M}_{\gamma} \beta^{\prime}$ to be continuous. Further, if we write

$\mathrm{KZ}_{3} \mathrm{X}_{3}{ }^{\prime}=\left(\mathrm{Z}_{1}+\mathrm{Z}_{2}\right)\left(\mathrm{X}_{1}{ }^{\prime}+\mathrm{X}_{2}{ }^{\prime}\right)=\mathrm{Z}_{1} \mathrm{X}_{1}{ }^{\prime}+\mathrm{Z}_{2} \mathrm{X}_{2}{ }^{\prime}+\left(\mathrm{Z}_{1} \mathrm{X}_{2}{ }^{\prime}+\mathrm{Z}_{2} \mathrm{X}_{1}{ }^{\prime}\right)$ a question of the independence of the original axd reflected waves in the first medium arises, $i . e$. the question whether

$$
\mathrm{Z}_{1} \mathrm{X}_{2}{ }^{\prime}+\mathrm{Z}_{2} \mathrm{X}_{1}{ }^{\prime}+\gamma_{1} \alpha_{2}{ }^{\prime}+\gamma_{2} \alpha_{1}^{\prime}=0 \text {. }
$$

This proves to be the case when the method of $\S 11$ is applied, and all the quantities are expressed in terms of $(\alpha, \beta, \gamma)$.

Now

$$
\begin{aligned}
\mathrm{KZXX}^{\prime}+\mathrm{M} \gamma \alpha^{\prime} & =\mathrm{KZZX}+\mathrm{M}_{\gamma \alpha}+\frac{1}{\mathrm{~V}}\{\mathrm{Z}(v \gamma-u \cdot \beta)+\gamma(w \mathrm{Y}-v \mathrm{Z})\} \\
& =\mathrm{K} Z \mathrm{ZX}+\mathrm{M}_{\gamma \alpha}+\frac{v}{\overline{\mathrm{V}}}(\mathrm{Y} \gamma-\mathrm{Z} \beta),
\end{aligned}
$$

when the aberrational forms

$$
\mathrm{X}^{\prime}=\mathrm{X}+\frac{\mathrm{M}}{\mu^{2}}(v \gamma-w \beta), \quad \alpha^{\prime}=a+\frac{\mathrm{K}}{\mu^{2}}(w \mathrm{Y}-v \mathrm{Z})
$$

are used. Similarly,

$$
\mathrm{KZY}^{\prime}+\mathrm{M}_{\gamma} \beta^{\prime}=\mathrm{KZY}+\mathrm{M}_{\gamma \beta}+\frac{w}{\bar{\nabla}}\left(\mathrm{Z}_{\alpha}-\mathrm{X}_{\gamma}\right) .
$$

When this is combined with the statement as to continuity there results

$$
\left\{(\mathrm{ZX}+\gamma \alpha)+\frac{w}{\mathrm{~V}}(\mathrm{Y} \gamma-\mathrm{Z} \beta)\right\}_{1}+\{\}_{2}=\left\{\mathrm{KZX}+\mathrm{M} \gamma \alpha+\frac{w}{\bar{V}}(\mathrm{Y} \gamma-\mathrm{Z} \beta)\right\}_{\mathrm{z}}
$$


The tangential stress-component is not balanced until the motional terms are taken into account. In the above they are $w \mathrm{P}_{1}, w \mathrm{P}_{2}$ on the left, and $\frac{w \mathrm{P}_{3}}{\mu^{2}}$ on the right hand. For the other component $\mathrm{Q}$ takes the place of $\mathrm{P}$.

$\$ 17$. The modification of the equations to include current $\left(i_{x} i_{y} i_{z}\right)$ treated as a line-discontinuity is simple. In the main we are concerned with the case of free æther, but for the present $K$ and $M$ are retained, and the work is written for the non-aberrational form, i. e., translation is relative to the dielectric. We have then, for the moving standpoint,

$$
\left.\begin{array}{rl}
\mathrm{K} \frac{d \mathrm{X}}{d t}+i_{x}=\mathrm{V}\left(\frac{d \gamma^{\prime}}{d y}-\frac{d \beta^{\prime}}{d z}\right), & \mathrm{M} \frac{d \alpha}{d t}=\mathrm{V}\left(\frac{d \mathrm{Y}^{\prime}}{d z}-\frac{d \mathrm{Z}^{\prime}}{d y}\right) \\
\mathrm{X}^{\prime}=\mathrm{X}+\mathrm{M}(v \gamma-w \beta) / \mathrm{V}, & \alpha^{\prime}=\alpha+\mathrm{K}(w \mathrm{Y}-v \mathrm{Z}) / \mathrm{V}
\end{array}\right\},
$$

with

$$
\rho=\mathrm{K} \Sigma \frac{d \mathrm{X}}{d x}, \text { and } \frac{d \rho}{d t}+\Sigma \frac{d i_{x}}{d x}=0 .
$$

In terms of letters without dash, we have, if $u v w$ are constant,

$$
\begin{aligned}
& \text { and } \\
& \frac{\mathrm{D}^{\prime}}{\mathrm{D} t} \equiv \frac{d}{d t}-u \frac{d}{d x}-v \frac{d}{d y}-w \frac{d}{d z}, \\
& \mathrm{~K} \frac{\mathrm{D}^{\prime} \mathrm{X}}{\mathrm{D} t}+p u+i_{x}=\mathrm{V}\left(\frac{d \gamma}{d y}-\frac{d \beta}{d z}\right), \quad \mathrm{M} \frac{\mathrm{D} \alpha}{\mathrm{D} t}=\mathrm{V}\left(\frac{d \mathrm{Y}}{d z}-\frac{d \mathrm{Z}}{d y}\right) .
\end{aligned}
$$

From the standpoint of the dielectric itself, these equations are

$$
\mathrm{K} \frac{d \mathrm{X}}{d t}+\rho u+i_{x}=\mathrm{V}\left(\frac{d \gamma}{d y}-\frac{d \beta}{d z}\right), \quad \mathrm{M} \frac{d \alpha}{d t}=\mathrm{V}\left(\frac{d \mathrm{Y}}{d z}-\frac{d \mathrm{Z}}{d y}\right) .
$$

The energy equation attaching to (53) contains terms in $i_{x} \ldots$ in addition to those found in $\S 11$; and we may also write those which result from supposing $u v w$ to depend on $t$. Thus

$$
\frac{d \mathrm{~S}}{d t}+\Sigma \mathrm{P} \frac{d u}{d t}+\Sigma i_{x} \mathrm{X}^{\prime}+\mathrm{V} \Sigma \frac{d}{d x}\left(\mathrm{Y}^{\prime} \gamma^{\prime}-\mathrm{Z}^{\prime} \beta^{\prime}\right)=0
$$

with a term for Joule's effect, and one combining the momentum of radiation with acceleration in translation.

But if we set out from (55), i. e. take the standpoint of the dielectric, and use $E$, we get with multipliers $X \ldots \alpha$,

$$
\frac{d \mathrm{E}}{d t}+\rho \Sigma u \mathrm{X}+\Sigma i_{x} \mathrm{X}=-\mathrm{V} \Sigma \frac{d}{d x}\left(\mathrm{Y}_{\gamma}-\mathrm{Z} \beta\right) .
$$


But $\quad \Sigma u X=\Sigma u X^{\prime}, \quad$ and

$\Sigma i_{x} \mathrm{X}=\Sigma i_{x} \mathrm{X}^{\prime}-\frac{\mathrm{M}}{\mathrm{V}} \Sigma i_{x}(v \gamma-w \beta)=\Sigma i_{x} \mathrm{X}^{\prime}+\frac{\mathrm{M}}{\mathrm{V}} \Sigma u\left(i_{y} \gamma-i_{z} \beta\right)$;

and with these the above becomes

$\frac{d \mathrm{E}}{d t}+\Sigma i_{x} \mathrm{X}^{\prime}+\rho \Sigma u \mathrm{X}^{\prime}+\frac{\mathrm{M}}{\mathrm{V}} \Sigma u\left(i_{y} \gamma-i_{x} \beta\right)=-\mathrm{V} \Sigma \frac{d}{d x}(\mathrm{Y} \gamma-\mathrm{Z} \beta)$.

The flux of energy to the unit-volume fixed in the dielectric accounts for (1) increase of E, (2) Joule's effect, and (3) the work done by electric and electromagnetic forces in virtue of the translation $(u v w)$. At first sight the use of multipliers $\mathrm{X}, \alpha$ instead of $\mathrm{X}^{\prime}, \alpha^{\prime}$ threatens difficulties as regards Joule's effect: these are resolved by the difference showing the work done by electromagnetic force, that work not appearing in (56), because the standpoint implied the translation.

If $\rho u^{\prime}, \ldots$ are written for $i_{x \ldots} \ldots,\left(u^{\prime} v^{\prime} z v^{\prime}\right)$ being a velocity additional to the general translation, Joule's effect is replaced by work done in virtue of the motion $\left(u^{\prime} v^{\prime} w^{\prime}\right)$, viz. $\rho \Sigma u^{\prime} \mathbf{X}^{\prime}$; and the conservation of charge is then expressed by

$$
\frac{d \rho}{d t}+\Sigma \frac{d}{d x}\left(\rho u^{\prime}\right)=0
$$

The mechanical side of electrical action may be separately shown by forming the time-rate of the momentum of radiation, that rate taken from the standpoint of the dielectric; that is, we start from (55) and form

$$
\frac{d \mathrm{R}}{d t} \text { or } \frac{d}{d t} \frac{\mathrm{KM}}{\mathrm{V}}\left(\mathrm{X} \beta-\mathrm{Y}_{\alpha}\right) \text {. }
$$

In the course of the work the terms

$$
\mathrm{M}_{\gamma} \Sigma \frac{d x}{d x} \text { and } \mathrm{Z}\left\{\mathrm{K} \Sigma \frac{d \mathrm{X}}{d x}-\rho\right\}
$$

are introduced, and the result is

$$
-\left(\frac{d \mathrm{Z}_{x}}{d x}+\frac{d \mathrm{Z}_{y}}{d y}+\frac{d \mathrm{Z}_{z}}{d z}\right)=\rho \mathrm{Z}^{\prime}+\frac{\mathrm{M}}{\mathrm{V}}\left(i \beta-i_{y} \alpha\right)+\frac{d \mathrm{R}}{d t} .
$$

That is, the rate of increase of (PQR) together with forces on charge and current (where they exist), is expressible as the body-force of a stress. Here the coefficients $\mathrm{K}$ and $\mathrm{M}$ occur in the stress, i.e.,

$\mathrm{Z}_{x}=-(\mathrm{KZX}+\mathrm{M} \gamma \alpha), \quad \mathrm{Z}_{x}=\frac{\mathrm{K}}{\mathrm{z}}\left(\mathrm{X}^{2}+\mathrm{Y}^{2}-\mathrm{Z}^{2}\right)+\frac{\mathrm{M}}{2}\left(\alpha^{2}+\beta^{2}-\gamma^{2}\right)$. 
An energy equation on the mechanical side is obtained by multiplying these by $(u v w)$, viz.,

$$
-\Sigma \frac{d}{d x}\left(u \mathbf{X}_{x}+v \mathbf{Y}_{x}+w \mathbf{Z}_{x}\right)=\rho \Sigma u \mathbf{X}^{\prime}+\frac{\mathrm{M}}{\overline{\mathrm{V}}} \Sigma u\left(i_{y} \gamma-i_{z} \beta\right)+\Sigma u \frac{d \mathrm{P}}{d t} .
$$

This equation is the complement of (56), and the division of $\frac{d}{d t} \Sigma \mathrm{P} u$ into two parts is noticeable. Equation (60) may also be got by subtracting (56) from (58), and quoting (37); but $\frac{\mathrm{DS}}{\mathrm{D} t}$ must be used in $(56)$ to transfer to the standpoint of (58).

$\$ 18$. We now pass to the special problem of discontinuities moving in free æther under circumstances which give a steady condition from the moving standpoint.

The solution of (54) has the form

$$
\begin{gathered}
\alpha=\frac{d \mathrm{H}}{d y}-\frac{d \mathrm{G}}{d z}, \quad \mathrm{X}=-\frac{d \psi}{z x}-\frac{1}{\mathrm{~V}} \frac{\mathrm{D}^{\prime} \mathrm{F}}{\mathrm{D} t} \\
a^{\prime}=a+\frac{1}{\mathrm{~V}}\left(v \frac{d \psi}{d z}-w \frac{d \psi}{d y}\right)+\frac{1}{\mathrm{~V}^{2}}\left(v \frac{\mathrm{D}^{\prime} \mathrm{H}}{\mathrm{D} t}-w \frac{\mathrm{D}^{\prime} \mathrm{G}}{\mathrm{D} t}\right), \\
\mathrm{X}^{\prime}=-\frac{1}{V} \frac{d \mathrm{~F}}{d t}-\frac{d}{d x}\left(\psi-\frac{1}{\mathrm{~V}} \Sigma \mathrm{F} u\right),
\end{gathered}
$$

where $\psi \mathrm{F} \mathrm{G} \mathrm{H}$ are defined by

$$
\nabla^{2} \Psi-\frac{1}{\mathrm{~V}^{2}} \frac{\mathrm{D}^{\prime 2} \psi}{\mathrm{D} t^{2}}+\rho=0, \quad \nabla^{2} \mathrm{~F}-\frac{1}{\mathrm{~V}^{2}} \frac{\mathrm{D}^{\prime 2} \mathrm{~F}}{\mathrm{D} t^{2}}+\frac{, u+i_{x}}{\mathrm{~V}}=0,
$$

subject to

In these we write

$$
\frac{1}{\mathrm{~V}} \frac{\mathrm{D}^{\prime} \psi}{\mathrm{D} t}+\Sigma \frac{d \mathrm{~F}}{d x}=0
$$

$$
\frac{d}{d t}=0, \quad \text { or } \quad \frac{\mathrm{D}^{\prime}}{\mathrm{D} t}=-\left(u \frac{d}{d x}+v \frac{d}{d y}+w \frac{d}{d z}\right) .
$$

Thus

$$
\alpha=\frac{d \mathrm{H}}{d y}-\frac{d \mathrm{G}}{d z}, \quad \mathrm{X}^{\prime}=-\frac{d}{d x}(\psi-\Sigma p \mathrm{~F}), \quad .
$$

where $(p, q, r)=(u, v, w) / V$, and the other variables are given by $\alpha^{\prime}=\alpha+r \mathrm{Y}-q \mathrm{Z}$, and $\mathrm{X}^{\prime}=\mathrm{X}+q \gamma-r \beta$.

For the integration of $\int \mathrm{S} d \tau$, i.e. $\int\left(\frac{1}{2} \Sigma \mathrm{XX}^{\prime}+\frac{1}{2} \Sigma \alpha \alpha^{\prime}\right) d \tau$, the forms of $X^{\prime} \ldots$ and $\alpha \ldots$ as gradient and curl are essential. For a volume-distribution,

$$
\int S d \tau=\int \frac{1}{2} \rho(\psi-\Sigma p F) d \tau_{i}+\int \frac{1}{2 \mathrm{~V}} \Sigma i_{x} \mathrm{~F} d \tau_{i}
$$


and for a typical current $i_{x} d \tau_{i}=\sigma i d x, \sigma$ being a small crosssection. Now $F$ depends on $u \rho+i_{x}$, and we may separate the discontinuities by writing $\mathrm{F}=p \psi+\mathrm{F}_{0}$, and $\mathrm{F}_{\theta}$ then satisfies

$$
\nabla^{2} \mathrm{~F}_{0}-\left(p \frac{d}{d x}+q \frac{d}{d y}+r \frac{d}{d z}\right)^{2} \mathrm{~F}_{0}+i_{x} / \mathrm{V}=0 .
$$

The condition $\frac{1}{\tilde{\mathrm{V}}} \frac{\mathrm{D}^{\prime} \psi}{\mathrm{D} t}+\Sigma \frac{d \mathrm{~F}}{d x}=0$ becomes, when $\frac{d}{d t}=0$,

$$
p \frac{d \psi}{d x}+q \frac{d \psi}{d y}+r \frac{d \psi}{d z}=\Sigma \frac{d \mathrm{~F}}{d x},
$$

which gives $\Sigma \frac{d \mathrm{~F}_{0}}{d x}=0$. We have, then,

$$
\begin{aligned}
\int S d \tau & =\int \frac{1}{2} \rho\left[\psi\left(1-\Sigma p^{2}\right)-\Sigma p \mathrm{~F}_{0}\right] d \tau_{i}+\int \frac{1}{2 \mathrm{~V}} \Sigma i_{x}\left(\mathrm{~F}_{0}+p \psi\right) d \tau_{i} \\
& =\int \frac{1}{2} \rho \psi\left(1-\Sigma p^{2}\right) d \tau_{i}+\int \frac{1}{2 V} \Sigma i_{x} \mathrm{~F}_{0} d \tau_{i}+\int \frac{1}{2}\left[\psi \Sigma p i_{x}-\rho \Sigma p \mathrm{~F}_{0}\right] d \tau_{i} .
\end{aligned}
$$

In the first two terms each discontinuity is associated with its own potential. In the statical case an element of potential has the discontinuity in the numerator, and in the denominator a distance connecting two points

$$
r=\sqrt{\left(x-x^{\prime}\right)^{2}+\left(y-y^{\prime}\right)^{2}+\left(z-z^{\prime}\right)^{2}} .
$$

With such a formula, for a denominator belonging to elements at $(x y z),\left(x^{\prime} y^{\prime} z^{\prime}\right)$, the various sections contributed to the numerator by the last term of (64) are

$$
\rho^{\prime} \Sigma p i_{x}-\rho \Sigma p i_{x}{ }^{\prime}+\rho \Sigma p i_{x}{ }^{\prime}-\rho^{\prime} \Sigma p i_{x} ;
$$

and these are cancelled whether $\rho$ and $i_{x}$ exist at one place, or $\rho$ only at one place and $i_{x}^{\prime}$ at another.

The form of denominator for the motional potential (to be considered more fully later) is suggested by transforming the wave-surface $\Sigma x^{\prime 2}=V^{2} t^{2}$ to a moving standpoint $\Sigma(x+u t)^{2}=\mathrm{V}^{2} t^{2}$, and treating this as a quadratic in $t$, viz.,

$$
\left(\mathrm{V}^{2}-\Sigma u^{2}\right) t-\Sigma u x \mp \sqrt{\left(\mathrm{V}^{2}-\Sigma u^{2}\right) \Sigma x^{2}+(\Sigma u x)^{2}}=0
$$

or

$$
\left(1-\Sigma p^{2}\right) t-\Sigma p x \mp \sqrt{\left(1-\Sigma p^{2}\right) \Sigma x^{2}+(\Sigma p x)^{2}}=0 .
$$

If we call this $\chi=0$, and write $\mathrm{R}^{2}=\left(1-\Sigma p^{2}\right) \Sigma x^{2}+(\Sigma p x)^{2}$, it is readily verified that $\mathrm{R}^{-1} f(\chi)$ satisfies the differential equation $\frac{\mathrm{D}^{\prime 2}}{\mathrm{D} t^{2}} \psi=V^{2} \nabla^{2} \psi$; and this solution replaces the 
typieal form $r^{-1} f(r-\mathrm{V} t)$. The quantity $\mathrm{R}$ is the required denominator and for points $x y z$ and $x^{\prime} y^{\prime} z^{\prime}$,

$$
\mathrm{R}^{2}=\left(1-\Sigma p^{2}\right) \Sigma\left(x-x^{\prime}\right)^{2}+\left(\Sigma p\left(x-x^{\prime}\right)\right)^{2}
$$

$\mathrm{R}$ is not altered by interchanging $x$ and $x^{\prime}$ and also not altered by reversing the sign of $(p q r)$ or $(u v w)$. Hence with this denominator the last section of (64), that containing cross-products, disappears in integration: At the same time it would seem impossible to ignore the part of the scalar potential due to $i_{x} \ldots$, in determining the potential of any conductor which may be in question, unless $\Sigma p \mathrm{~F}_{0}=0$ or $\Sigma p i_{x}=0$. In that connexion this special case is important.

When the whole solution is expressed in terms of $\psi, \mathrm{F}_{0}$, $\mathrm{G}_{0}, \mathrm{H}_{0}$, it stands

$$
\left.\begin{array}{rl}
\alpha & =\frac{d \mathrm{H}_{0}}{d z}-\frac{d \mathrm{G}_{0}}{d z}+r \frac{d \psi}{d y}-q \frac{d \psi}{d z}, \quad-\mathrm{X}=\left(1-\Sigma p^{2}\right) \frac{d \psi}{d x}-\frac{d}{d x} \Sigma p \mathrm{~F}_{0} \\
-\mathrm{X} & =\left(1-p^{2}\right) \frac{d \psi}{d x}-p q \frac{d \psi}{d y}-p r \frac{d \psi}{d z}-\left(p \frac{d}{d x}+q \frac{d}{d y}+r \frac{d}{d z}\right) \mathrm{F}_{0} \\
\alpha^{\prime} & =\left(-p q \frac{d}{d x}+\overline{1-q^{2}} \frac{d}{d y}-q r \frac{d}{d z}\right) \mathrm{H}_{0}-\left(-p r \frac{d}{d x}-q r \frac{d}{d y}+\overline{1-r^{2}} \frac{d}{d z}\right) \mathrm{G}_{0}
\end{array}\right\}
$$

in which form the æolotropic character of each solution plainly appears.

$\S 19$. There is an important difference in the energies attached to the two solutions, the scalar solution with $\rho, \psi$ and the vector solution with $i_{x} \ldots \mathrm{F}_{0} \ldots$ In the solution for charge $\rho$ essentially determines $\mathrm{X}$... (in a dielectric $\mathrm{KX}, \ldots$ ), though the motional form is given by the intervention of other variables. The solution is therefore momentil in character, and for it we write

$$
\mathrm{P}=-\frac{d \mathrm{~S}}{d u}, \quad \text { and } \quad \mathrm{E}=\mathrm{S}-\Sigma u \frac{d \mathrm{~S}}{d u}=\mathrm{S}+\mathrm{T} .
$$

In the solution for current $i_{x} \ldots$ essentially determine $\alpha^{\prime} \ldots$, that is quantities with the character of velocities, and for this solution we write

$$
\mathrm{P}^{\prime}=\frac{d \mathrm{~S}^{\prime}}{d u}, \quad \text { and } \quad \mathrm{E}^{\prime}=\mathrm{S}^{\prime}+\Sigma u \frac{d \mathrm{~S}^{\prime}}{d u}=\mathrm{S}^{\prime}+\mathrm{T}^{\prime} .
$$

The various quantities refer to integrated results. The distinction is valid apart from any translation, i.e. there is always a distinction between $K \mathbf{X}$ and $\mathbf{X}$ or between $\mathrm{M}_{\alpha}$ and $a$, although it may be lost sight of when $K$ and $M$ are each written $=1$.

If we consider the charge alone, we have $a^{\prime}=\beta^{\prime}=\gamma^{\prime}=0$; 
but $\Sigma X X X^{\prime}-\Sigma \alpha a^{\prime}=\Sigma X^{2}-\Sigma \alpha^{2}$ always, and therefore in this case $\frac{1}{2} \Sigma X X^{\prime}=\frac{1}{2} \Sigma X^{2}-\frac{1}{2} \Sigma \alpha^{2}$, or integrating we have $S=E_{e}-E_{m}$. But $\mathrm{E}=\mathrm{E}_{\imath}+\mathrm{E}_{m}$, and therefore

$$
\mathrm{E}_{e}=\mathrm{S}+\frac{1}{2} \mathrm{~T} \text {, and } \mathrm{E}_{m}=\frac{1}{2} \mathrm{~T} \text {, }
$$

i.e., the integral values of $\mathrm{E}_{e}$ and $\mathbf{E}_{m}$ are separately deduciblo from that of $\mathrm{S}$. If we consider the current solution alone, $\Sigma X X^{\prime}$ is not $=0$ at each point of the field unless $\Sigma p i_{x}=0$; but its integral vanishes because the divergence of ( $\mathrm{X} \mathrm{Y} \mathrm{Z}$ ) for this solution is

$$
\left(p \frac{d}{d x}+q \frac{d}{d y}+r \frac{d}{d z}\right) \Sigma \frac{d \mathrm{~F}_{0}}{d x} \text {, and } \Sigma \frac{d \mathrm{~F}_{0}}{d x}=0 .
$$

With $\int \Sigma X^{\prime} d \tau=0$ goes $\int \frac{1}{2} \Sigma \alpha \alpha^{\prime} d \tau=\int\left(\frac{1}{2} \Sigma \alpha^{2}-\frac{1}{2} \Sigma X^{2}\right) d \tau$, i.e., $\mathrm{S}^{\prime}=\mathrm{E}_{m}^{\prime}-\mathrm{E}_{e}^{\prime}$,

$$
\mathrm{E}_{m}^{\prime}=\mathrm{S}^{\prime}+\frac{1}{2} \mathrm{~T}^{\prime} \text {, and } \mathrm{E}_{e}^{\prime}=\frac{1}{2} \mathrm{~T}^{\prime} . . .
$$

Before proceeding to the general solution for any ellipsoid, the simple case which corresponds to a sphere in statical potentials may be briefly noticed. With $i_{x}=i_{y}=i_{z}=0$

$$
\begin{aligned}
-\mathrm{X}^{\prime} & =\frac{d \psi}{d x}\left(1-\Sigma p^{2}\right), \quad-\mathrm{X}=\left(1-p^{2}\right) \frac{d \psi}{d x}-p q \frac{d \psi}{d y}-p r \frac{d \psi}{d z} \\
\alpha^{\prime} & =0, \quad \alpha=r \frac{d \psi}{d y}-g \frac{d \psi}{d z} ; \quad \rho=\Sigma \frac{\mathrm{X}}{d x} .
\end{aligned}
$$

For $\psi=C / R$ the surface $R=R_{0}$ is an equipotential surface, and may be treated as a conductor. We find $\mathrm{X}=\mathrm{C} x\left(1-\Sigma p^{2}\right) / \mathrm{R}^{3}$, and if $e$ is the total charge,

$$
e=\int \Sigma l \mathrm{X} d \mathrm{~S}=\frac{\mathrm{C}\left(1-\Sigma p^{2}\right)}{\mathrm{R}_{0}{ }^{3}} \int \Sigma l x d \mathrm{~S}=\frac{3 \mathrm{C} \tau_{0}\left(1-\Sigma p^{2}\right)}{\mathrm{R}_{0}{ }^{3}}=4 \pi \mathrm{C},
$$

since the polar and equatorial semi-axes are $R_{0}$ and $\mathbf{R}_{0} / \sqrt{1-\Sigma p^{2}}$ respectively. Thus

$\psi=\frac{e}{4 \pi \mathbf{R}}$, and then $S=\frac{e}{2} \cdot \psi_{s}\left(1-\Sigma p^{2}\right)=\frac{e^{2}\left(1-\Sigma p^{2}\right)}{8 \pi \mathbf{R}_{0}}$.

The potential is properly $\phi=\psi\left(1-\Sigma p^{2}\right)=e\left(1-\Sigma p^{2}\right) / 4 \pi \mathrm{R}$, which makes $\mathrm{X}^{\prime}=-\frac{d \phi}{d x}$. For a uniform volume distribution $\phi_{i}=\frac{\rho}{2}\left(\mathrm{R}_{0}{ }^{2}-\frac{1}{3} \mathrm{R}^{2}\right), \quad \phi_{\theta}=\rho \mathrm{R}_{0}{ }^{3} / 3 \mathrm{R}$; and the integration $\int \frac{1}{2} \rho \phi_{i} d \tau_{i}$ for $\mathrm{S}$ yields $3 e^{2}\left(1-\Sigma p^{2}\right) / 20 \pi \mathrm{R}_{0}$.

Thns the typical elementary solution which corresponds to that of the sphere in attractions here belongs to a particular oblate spheroid. 